\title{
Turbulence structures and statistics of a supersonic turbulent boundary layer subjected to concave surface curvature
}

\begin{tabular}{|c|c|}
\hline Journal: & Journal of Fluid Mechanics \\
\hline Manuscript ID & JFM-18-S-0662.R2 \\
\hline mss type: & JFM Papers \\
\hline $\begin{array}{r}\text { Date Submitted by the } \\
\text { Author: }\end{array}$ & 20-Dec-2018 \\
\hline Complete List of Authors: & $\begin{array}{l}\text { Sun, Mingbo; Science and Technology on Scramjet Lab } \\
\text { Sandham, Neil; University of Southampton, Aeronautics and } \\
\text { Astronautics, School of Engineering Sciences; Dept. of Aeronautics + } \\
\text { Astronautics Engineering, School of Engineering Sciences } \\
\text { Hu, Zhiwei; University of Southampton, SES-Aero }\end{array}$ \\
\hline Keyword: & $\begin{array}{l}\text { High-speed flow }<\text { Aerodynamics, Boundary layer structure }<\text { Boundary } \\
\text { Layers, Compressible boundary layers }<\text { Compressible Flows }\end{array}$ \\
\hline
\end{tabular}

\section{SCHOLARONE Manuscripts}




\title{
Turbulence structures and statistics of a supersonic turbulent boundary layer subjected to concave surface curvature
}

\author{
M. B. Sun ${ }^{1}{ }^{\dagger} \dagger$, N.D. Sandham ${ }^{2}$, Z. W. Hu ${ }^{2}$ \\ ${ }^{1}$ Science and Technology on Scramjet Laboratory, National University of Defense Technology, \\ Changsha, 410073, China \\ ${ }^{2}$ Aerodynamics and Flight Mechanics, Faculty of Engineering and the Environment, University \\ of Southampton, Southampton, SO17 1BJ, UK
}

(Received xx; revised xx; accepted $\mathrm{xx}$ )

Supersonic turbulent flows at Mach 2.7 over concave surfaces for two different curvature radii were investigated and compared with a flat plate turbulent boundary layer using direct numerical simulations. The streamwise velocity reduces in the outer part of the boundary layer due to the compression while it increases near the wall due to curvature, with a higher shape factor for the concave cases. The near wall spanwise streak spacing reduces compared to the flat plate, with large scale streaks and turbulence amplification also observed. Streamwise velocity iso-surfaces and streamlines show the generation of Görtler-like vortices, consistent with significant centrifugal effects. Abundant small vortices are shown to be associated with large baroclinic production of vorticity that is caused by the density and pressure gradients that are associated with the concave compression. Profiles of turbulent kinetic energy (TKE) and turbulent Mach number exhibit a characteristic two-layer structure in the concave boundary layer cases. In the outer layer, turbulence is greatly amplified, whereas a local balance exists in the inner layer. Turbulent energy budget analysis shows that both production and dissipation increase near the concave wall, whereas in the outer part of the boundary layer, the production is increased and ultimately balanced by convection and turbulent transport.

Key words: Turbulence structures, Supersonic turbulent boundary layer, Concave surface

\section{Introduction}

Supersonic boundary layers have been of great practical interest to researchers for several decades. While the majority of the research has been focused on flat geometries, realistic high-speed (e.g., supersonic and hypersonic) vehicles are composed of curved surfaces and more complex geometries. Concave surface curvature can introduce significant distortion to a compressible boundary layer flow due to multiple, potentially coupled, effects including adverse pressure gradient (APG), bulk flow compression, and possible centrifugal instabilities (see White 2007; Saric 1994; Smits \& Dussauge 2006, for examples). Improved understanding of the physical processes driving these highspeed turbulent boundary layer flows is necessary to design more efficient supersonic

$\dagger$ Email address for correspondence: sunmingbo@nudt.edu.cn 
and hypersonic vehicles, which may be able to utilize the physical mechanisms to their advantage.

Several experimental and theoretical studies have been performed to gain insight into these processes, as reviewed in Spina et al. (1994), showing that APGs and concave wall curvature destabilize the boundary layer and enhance turbulent mixing, explained by Green (1970) in terms of the conservation of angular momentum. The destabilization physically manifests itself as an increase in turbulent fluctuations due to the streamline curvature, APGs and bulk compression. This is in contrast to the stabilizing effects of favourable pressure gradients on a boundary layer and the weakening of the coherent structures (Spalart \& Watmuff (1993); Tichenor et al. (2013); Sun et al. (2017)), which leads to a turbulence reduction in the outer region of the boundary layer.

Experiments by Donovan et al. (1994) in a Mach 2.9 turbulent flow showed how the wall shear stress increases over a concave wall. Fernando \& Smits (1990) investigated a flat plate with an imposed pressure gradient equal to that over a curved ramp and an augmentation in the wall friction was reported, highlighting the importance of the curvature. Smith \& Smits $(1994,1995)$ did experiments to compare the Reynolds stresses of the curved flow with a flat plate flow which has an equal adverse pressure gradient. Flaherty \& Austin $(2013 a)$ also demonstrated that heat transfer was also significantly enhanced on concave surfaces.

Spina et al. (1994) used two parameters to characterize the dilatation/compression and the streamline curvature. The bulk dilatation or compression impulse parameter is defined as $I_{p}=\gamma^{-1} \ln \left(p_{2} / p_{1}\right)$, where $\gamma$ is the specific heat ratio with $p_{1}$ and $p_{2}$ the static pressure before and after the pressure gradient, respectively. The parameter $I_{\varphi}=\Delta \varphi$ represents the change in the wall angle in radians. For a Mach 2.7 case with $I_{p}=0.35$ and $I_{\varphi}=0.1$, Luker et al. (1998) observed a 70-100\% increase in the turbulent stress levels. Smith \& Smits $(1994,1995)$ found higher levels of turbulent stresses when the pressure gradient was applied over smaller distances. They noted the peak Reynolds shear stress was doubled for $I_{p}=0.46$ and $I_{\varphi}=0.14$ over a distance of 7 times boundary layer thickness. Recently Neel et al. (2016) studied the effects of a streamline curvatureinduced APG on a Mach 4.9 turbulent boundary layer with parameters $I_{p}=0.47$ and $I_{\varphi}=0.13$, which revealed a large amplification of the shear stresses in the boundary layer over the APG region, where schlieren images and averaged velocities from planar PIV revealed a thinning of the boundary layer.

Over concave surfaces, it is possible to excite an instability mode due to centrifugal effects. This instability manifests itself as streamwise-oriented counter-rotating vortices known as Görtler vortices (Görtler 1954). A relevant non-dimensional parameter is the local Görtler number defined as $G_{\theta}=\operatorname{Re}_{\theta} \sqrt{\theta / R}$, where $\theta$ is the boundary layer momentum thickness, $\operatorname{Re}_{\theta}$ is the Reynolds number based on the displacement thickness and freestream velocity. $R$ is the surface radius of curvature. In general, as the Görtler number increases, Görtler vortices become more pronounced. Görtler vortices can form even in fully-developed turbulent flows. For supersonic curved boundary layers, as proposed by Bradshaw (1974), the destabilizing process is caused by the combined effects of the streamline curvature $(\partial V / \partial x)$, the normal $(\partial p / \partial y)$ and streamwise $(\partial p / \partial x)$ pressure gradients, and the bulk dilatation $(\nabla \cdot \vec{V})$, making it extremely complicated. Theoretical work has also been conducted to analyze the instability mode and the stability limit leading to the Görtler vortices, as summarized in the review by Spina et al. (1994). Recently, Ren \& Fu (2015b) and Li et al. (2010) ran simulations for laminar flow to characterize the formation of Görtler vortices and deduced that secondary mode 
instabilities result in sinusoidal oscillations in the Görtler vortices, and eventually lead to vortex breakdown and transition to turbulence.

It has been suggested that the increase in large-scale activity leads to an overall increase of the turbulent intensity(Harun et al. 2013). Several experiments have tried to visualize the flow structures of Görtler vortices, including Luca et al. (1993) for a Mach 7 flow, Ciolkosz \& Spina (2006) at low supersonic Mach numbers varying from 1.06 to 2.87 and Flaherty (2013) at Mach 5.12. Recently, Wang \& Wang (2016) studied the response of turbulent structures in a $M a=2.95$ supersonic boundary layer to concave curvature experimentally and found that the large scale vortices formed in the flat plate region break down into smaller ones immediately after being convected into the concave region. Since only a 2D longitudinal slice was obtained, it was difficult to speculate about the $3 \mathrm{D}$ vortex structures on the concave walls.

Experiments are limited in terms of what can be actually measured. Additionally, there are limited publications (Tong et al. 2017) on numerical simulations of supersonic flow over curved surface that could give more insight. In this paper, we present direct numerical simulation (DNS) studies of supersonic Mach 2.7 flows over concave surfaces with different curvature radius $R=308 \mathrm{~mm}, 908 \mathrm{~mm}$ and a flat plate. The parameters defined by Spina et al. (1994) are $I_{p}=0.293$ and $I_{\varphi}=0.055$ for $\mathrm{R}=908 \mathrm{~mm}$, while $I_{p}=0.822$ and $I_{\varphi}=0.165$ for $R=308 \mathrm{~mm}$, for which significant curvature effects are expected. The detailed turbulence structures and flow statistics will be analyzed. The paper is organized as follows. In Section 2 the numerical method and the simulation parameters are introduced. Section 3 discusses turbulence statistics such as mean profiles, boundary layer thickness, shape factors and pressure fluctuations, while Section 4 discusses three dimensional turbulent structures in concave flows. Statistical quantities such as turbulence fluctuations, turbulent kinetic energy (TKE) and budget are compared with the flat plate case in Section 5. Finally, conclusions are given in Section 6.

\section{Computational setup}

\subsection{Numerical simulation and turbulent inflow generation}

All simulations in this paper solve the three-dimensional unsteady compressible NavierStokes equations directly without any modeling, using an in-house DNS code. The code has been previously used for studies of instability, transition and turbulent high-speed flows (Sandham 2016; Sandham et al. 2014). Here we provide only a brief recap of the main features of the code. Details of the governing equations and algorithm can be found in Touber \& Sandham (2009) and the references cited therein.

The governing equations are solved in non-dimensionalized conservative forms of the continuity, momentum, and energy equations in curvilinear coordinates $(\xi, \eta, \zeta)$ which are transformed from Cartesian coordinates $(x, y, z)$. The equations are non-dimensionalized by the inflow parameters.

$$
\frac{\partial U}{\partial t}+\frac{\partial F}{\partial \xi}+\frac{\partial G}{\partial \eta}+\frac{\partial H}{\partial \zeta}=0
$$

The conservative variables and the flux terms are given by

$$
U=J\left\{\begin{array}{l}
\rho \\
\rho u \\
\rho v \\
\rho w \\
\rho e
\end{array}\right\} \text { and } F=F_{c}+F_{v}=J r_{\xi}\left\{\begin{array}{l}
\rho u^{*} \\
\rho u u^{*}+p s_{x} \\
\rho v u^{*}+p s_{y} \\
\rho w u^{*}+p s_{z} \\
(\rho e+p) u^{*}
\end{array}\right\}-J r_{\xi}\left\{\begin{array}{l}
0 \\
\sigma_{x x} s_{x}+\sigma_{x y} s_{y}+\sigma_{x z} s_{z} \\
\sigma_{y x} s_{x}+\sigma_{y y} s_{y}+\sigma_{y z} s_{z} \\
\sigma_{z x} s_{x}+\sigma_{z y} s_{y}+\sigma_{z z} s_{z} \\
\tau_{x} s_{x}+\tau_{y} s_{y}+\tau_{z} s_{z}
\end{array}\right\}
$$


where

$$
\begin{aligned}
& s_{x}=\xi_{x} / r_{\xi} \\
& u^{*}=u s_{x}+v s_{y}+w s_{z}, r_{\xi}=\sqrt{\xi_{x}^{2}+\xi_{y}^{2}+\xi_{z}^{2}} \\
& \tau_{x}=\sigma_{x x} u+\sigma_{x y} v+\sigma_{x z} w-q_{x} \\
& \tau_{y}=\sigma_{y x} u+\sigma_{y y} v+\sigma_{y z} w-q_{y} \\
& \tau_{z}=\sigma_{z x} u+\sigma_{z y} v+\sigma_{z z} w-q_{z}
\end{aligned}
$$

Here, $F_{c}$ and $F_{v}$ denotes the convective and viscous term, respectively. The flux terms $G$ and $H$ have similar forms as $F$. The viscous stress and heat flux terms are obtained from Newtonian and Fourier models, given by

$$
\sigma_{i j}=\frac{2 \mu}{\operatorname{Re}}\left[\frac{1}{2}\left(\frac{\partial u_{i}}{\partial x_{j}}+\frac{\partial u_{j}}{\partial x_{i}}\right)-\frac{1}{3} \frac{\partial u_{k}}{\partial x_{k}} \delta_{i j}\right]
$$

and

$$
q_{j}=-\frac{\mu}{(\gamma-1) \operatorname{Re} \operatorname{Pr} M_{\infty}^{2}} \frac{\partial T}{\partial x_{j}}
$$

The viscosity $\mu$ is calculated from the Sutherlands law, and the relationship between the thermodynamic variables is given by the ideal gas state equation, i.e.

$$
\mu(T)=T^{\frac{3}{2}} \frac{1+T_{s} / T_{\infty}}{T+T_{s} / T_{\infty}}
$$

and

$$
p=\frac{\rho T}{\gamma M_{\infty}^{2}}
$$

where $T_{s}=110.4$ is the Sutherland constant for air. For all the numerical simulations carried out in this work $\operatorname{Pr}=0.72$ and $\gamma=1.4$ have been used.

The code solves the compressible Navier-Stokes equations using an entropy-splitting approach for the Euler terms and fourth-order accurate finite differences. A third order Runge-Kutta scheme is used for matching in time. The digital filter approach of Xie \& Castro (2008) is employed at the inflow, which promotes the development of the flow to fully turbulent conditions. A detailed description of the optimized digital filter used in this paper can be found in Touber (2010). This method is robust to the choice of length scales, as long as the prescribed length scales are at least as large as the integral length scale of the flow and adequate distance is provided downstream to allow the turbulence to develop (Wang et al. 2015). In the present study, the streamwise characteristic length scales for the three velocity components $u, v$ and $w$ are set to $0.65 \delta_{i}, 0.35 \delta_{i}$ and $0.35 \delta_{i}\left(\delta_{i}\right.$ denotes the $99 \%$ boundary-layer thickness at the inflow), respectively. Mean and rootmean-square (RMS) profiles are prescribed beforehand for the wall boundary layers. The mean inflow profile is generated using the same approach as Touber \& Sandham (2009). The inflow RMS values are taken from the DNS results of Schlatter \& Örlü (2010) for a similar Reynolds number.

The inflow parameters (given in Table 1) are set in accordance with the Mach 2.7 experiments of Sun et al. (2013) and the recent simulations of Sun \& $\mathrm{Hu}(2018 a, b, c)$. The bottom wall $99 \%$ boundary-layer thickness, which is the same for all simulations, is estimated to be $\delta_{i}=5.7 \mathrm{~mm}$, which gives the compressible (including density variations) boundary-layer displacement thickness $\delta_{i}{ }^{*}=1.96 \mathrm{~mm}$ and momentum thicknesses $\theta=0.41 \mathrm{~mm}$, respectively, and corresponding Reynolds numbers $R e_{\delta *}=17,213, R e_{\theta}=3,600$. A full list of the cases conducted in the present study is given in Table 2. 


$\begin{array}{lccccc}\text { Mach number } & \text { Stagnation } & \text { Stagnation } & \text { BL 99\% } & \text { Momentum } & \text { Reynolds } \\ & \text { temperature } & \text { pressure } & \text { thickness } & \text { thickness } & \text { number } \\ M a & T_{0} & P_{0} & \delta_{i} & \theta & R e_{\theta} \\ 2.7 & 300 \mathrm{~K} & 100 \mathrm{kPa} & 5.7 \mathrm{~mm} & 0.41 \mathrm{~mm} & 3600\end{array}$

TABLE 1. Flow conditions for the simulations, including the dimensional boundary-layer (BL) thicknesses and Reynolds number at the inflow.

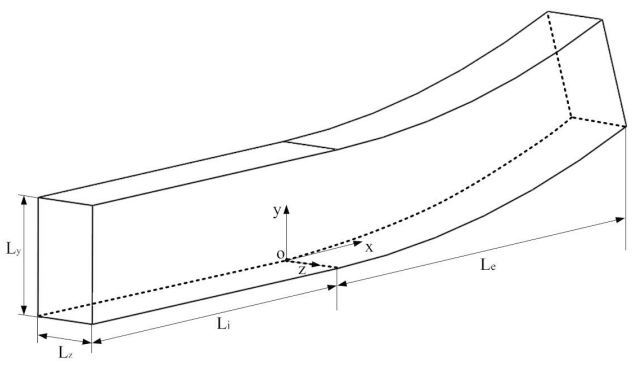

(a) sketch of the flat and concave domain

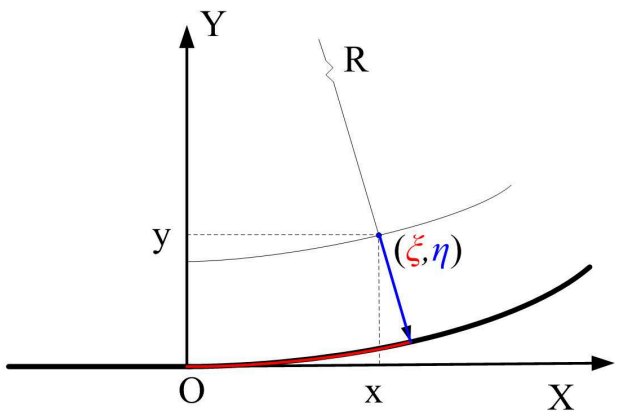

(b) sketch of the concave curvature radii

FIGURE 1. Sketch of the domain used for the simulations

\subsection{Domain and grid distribution}

Although the use of a digital filter to generate the inflow boundary condition significantly reduces the length required for boundary-layer development, there should nevertheless be enough distance to allow the boundary layers to adjust to an equilibrium state before the turning point (Xie \& Castro 2008). It was found by Touber (2010) that a distance of 12 times the inflow boundary-layer thickness is enough to obtain realistic turbulent mean and RMS profiles from the digital filter inflow generator used, whereas Bradshaw (1974) suggested that the recovery length is usually ten times of the incoming boundary layer thickness. Here we choose $L_{i}=15$ and $\delta_{i} \approx 85 \mathrm{~mm}$ for a fully developed turbulence generation. The boundary layer thickness at the point of the corner is denoted as $\delta_{0}$. An evaluation of the boundary layer thickness shows $\delta_{0} \approx 6.19 \mathrm{~mm}$. The concave length $L_{e}$ is set to $L_{e} \geqslant 10 \delta_{0}$. In this study, we consider two concave curvature radii, $908 \mathrm{~mm}$ and $308 \mathrm{~mm}$, and we take $L_{e}=16 \delta_{0} \approx 100 \mathrm{~mm}$ and the total length of the computational domain is $L_{x}=185 \mathrm{~mm}$. For brevity, the two concave cases are denoted as CurvR908mm and CurvR308mm for curvature radii of $908 \mathrm{~mm}$ and $308 \mathrm{~mm}$, respectively. A sketch of the computational domain is shown in figure 1. A fixed coordinate system $(x, y, z)$ is used, denoting the streamwise, bottom wall-normal and spanwise directions, respectively, for the straight wall segment. The origin is set at the turning point, which is $85 \mathrm{~mm}$ downstream of the inlet. Thus the inlet plane is located at $x=-85 \mathrm{~mm}$. The curvilinear coordinates $(\xi, \eta, \zeta)$ in the concave region are transformed from the cartesian coordinate system $(x, y, z)$ with $\xi=R \arctan \left(\frac{x}{R-y}\right), \eta=R-r=R-\sqrt{(R-y)^{2}+x^{2}}$ and $\zeta=z$. Thus the coordinate transformation coefficients $\left(r_{\xi}, r_{\eta}\right.$ and $\left.r_{\zeta}\right)$ are known for all cases.

A stretched grid is used near the wall in the $y$ direction, changing gradually to a uniform grid outside the boundary layer with a prescribed spacing. Grid smoothness is very important, especially when high-order schemes are used. Two grid stretching 


$\begin{array}{lcccc}\text { Case } & \text { Domain size } & \text { Grid number } & \text { Grid control } & \text { Grid spacing } \\ & L_{x} \times L_{y} \times L_{z} \mathrm{~mm} & N_{x} \times N_{y} \times N_{z} & \nu_{b}(\mathrm{~mm}), N_{b}, \beta & \Delta_{x}^{+} \Delta_{y}^{+} \Delta_{z}^{+} \\ \text {All cases } & 185 \times 25 \times 15 & 2305 \times 241 \times 289 & 1.85,81,1.80 & 6.50 .8-9.84 .1\end{array}$

TABLE 2. Grid numbers and stretching control parameters for different cases

functions with at least second-order continuity at the interface are combined to give coordinates $y_{i}$ in the direction normal to the bottom wall as

$$
y_{i}=\left\{\begin{array}{l}
y_{b} \frac{\sinh \left(\beta \eta_{j}\right)}{\sinh (\beta)}, \quad \eta_{j}=\frac{j-1}{N_{b}-1}, \quad j \leqslant N_{b} \\
a \cdot j^{5}+b \cdot j^{3}+c \cdot j+y_{\text {mid }},
\end{array}\right.
$$

where $y_{\text {mid }}$ is the middle of the domain, while the parameters $a, b$ and $c$ are fixed by continuity considerations up to second order. A predefined distance from the wall, $y_{b}$, is used to specify the region for grid stretching, with $N_{b}$ the number of grid points inside this region. A fifth-order polynomial enables the grid to change quickly from a stretched grid near the wall to an almost uniform grid away from the wall.

In the $y$ direction, the grid spacing is $y_{1}^{+}=0.8$ for the first point off the wall and $\Delta y_{\max }^{+}=9.8$ in the centre of the domain (all wall units are calculated based on the inflow friction velocity). The streamwise direction has a uniform grid distribution, with $\Delta x^{+}=6.5$. In the spanwise direction, the grid spacing is also uniform and fixed at $\Delta z^{+}=4.1$. The grid suitability for the present simulations was also verified by changing the grid resolution in all directions. A sensitive feature was found to be the peak skin friction in the vicinity of the outlet on concave surface which varied by less than $5.0 \%$, for a grid that was coarsened by $50 \%$ in all directions. A variant of the standard total variation diminishing scheme is employed for strong discontinuity capturing (Yee et al. 1999), which is turned off within boundary layers by incorporating the Ducros sensor (Ducros et al. 1999). Comparison of mean profiles of flat plate with DNS results of Schlatter \& Örlü (2010) were made and found to be consistent (Sun et al. 2017). The boundary layer thickness at $x=0 \mathrm{~mm}$ is estimated as $\delta_{0} \approx 6.19 \mathrm{~mm}$ and the compressible boundary-layer displacement and momentum thicknesses are $\delta_{0}{ }^{*}=2.37 \mathrm{~mm}$ and $\theta_{0}=$ $0.48 \mathrm{~mm}$, respectively.

No-slip boundary conditions were enforced on the bottom wall (shown in figure 1). The wall temperature is fixed at a value equal to the stagnation temperature of the inflow. Integrated characteristic boundary conditions (Thompson 1990) are used at the outflow and the top boundary. Periodic boundary conditions are applied in the spanwise direction.

\subsection{Grid and numerical validity}

Statistical data are based on averaging flowfields over 360 non-dimensional time units $\left(\delta_{0} / U_{\infty}\right)$ after running the simulations for 240 non-dimensional units to let the flow develop. To check the spanwise domain size, two-point spanwise correlations of velocity perturbations at different wall normal locations are given in figure $2(\mathrm{a}, \mathrm{b})$. The correlation definition is the same as in Pirozzoli et al. (2004). As shown, the correlation coefficients decay rapidly to zero with the increase in the spanwise separation. It is clear that the correlation coefficients reduce to zero well within the domain, suggesting that the simulation domain is sufficiently wide to resolve the turbulence dynamics. The adequacy of the spatial resolution is also confirmed by examining the one-dimensional energy 


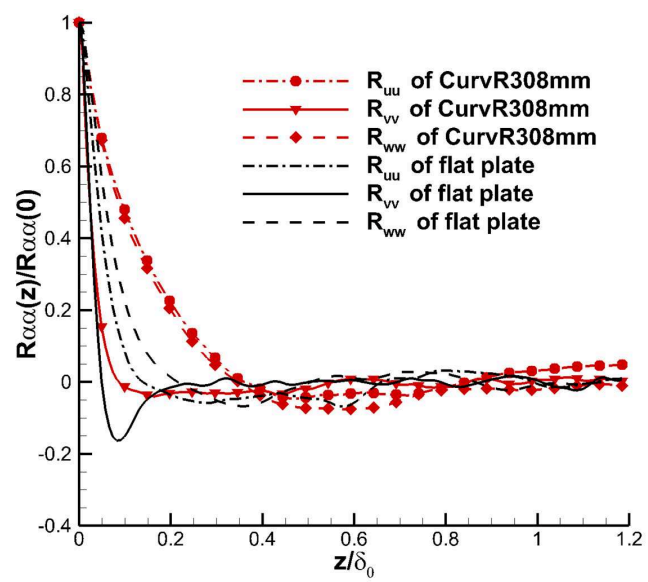

(a) two-point correlation at $y / \delta_{0}=0.0587$

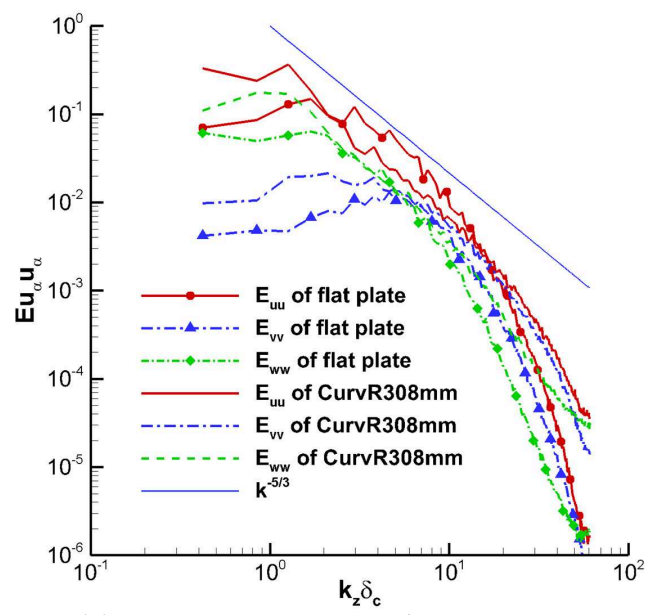

(c) energy spectra at $y / \delta_{0}=0.0587$

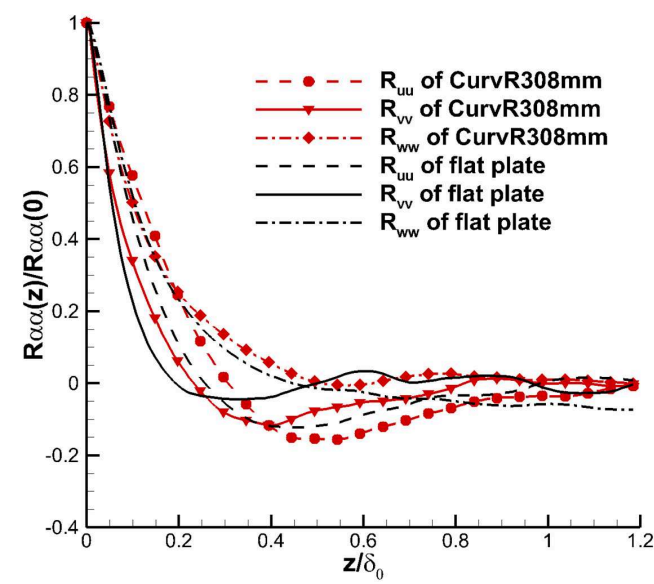

(b) two-point correlations at $y / \delta_{0}=0.2908$

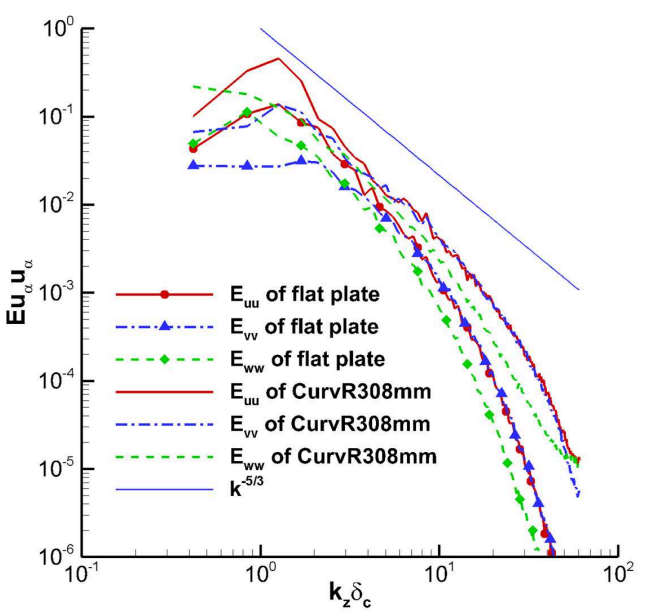

(d) energy spectra at $y / \delta_{0}=0.2908$

Figure 2. Two-point correlation and energy spectra of the velocity components at different wall normal locations $x / \delta_{0}=11.5$

spectra (Pirozzoli et al. 2004), as shown in figure 2(c,d), where $E u_{a} u_{a}$ is the energy spectrum for the velocity component. It can be seen that all spectra exhibit a drop off of at least four order of magnitudes. Compared to the DNS result of similar flows (Guarini et al. 2000), these spectra suggest the resolution is adequate.

Turbulent boundary-layer mean velocity profiles and distributions of RMS values at $x=0 \mathrm{~mm}$ on the flat plate (at the concave turning point) are evaluated. The mean inflow profile is generated using the same approach as Touber \& Sandham (2009). The inflow RMS values are taken from the DNS results of Schlatter \& Örlü (2010) for a similar Reynolds number. A compressible scaling, as suggested by Morkovin (1962) and validated by Duan \& Beekman (2011) for high Mach numbers, is used to transform the incompressible RMS values. The calculated compressible boundary-layer displacement and momentum thicknesses at $x=0 \mathrm{~mm}$ are $\delta_{0}{ }^{*}=2.37 \mathrm{~mm}, \theta_{0}=0.48 \mathrm{~mm}$, respectively. It should be noted that the Reynolds number based on the freestream flow properties and the momentum thickness, i.e. $\operatorname{Re}_{\theta}=\rho_{e} U_{e} \theta / \mu_{w}$, is 4,215. The corresponding Reynolds number under the van Driest scaling, i.e. $\operatorname{Re}_{\theta, v d}=\rho_{w} U_{e}^{v d} \theta^{v d} / \mu_{w}$, is 1,078 . The subscripts e and $\mathrm{w}$ above denote the main flow and wall parameters, respectively, 


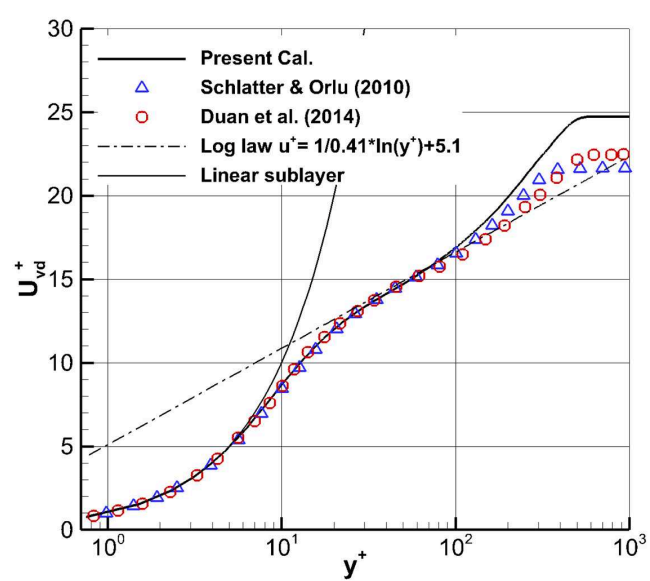

(a) Mean velocity distribution

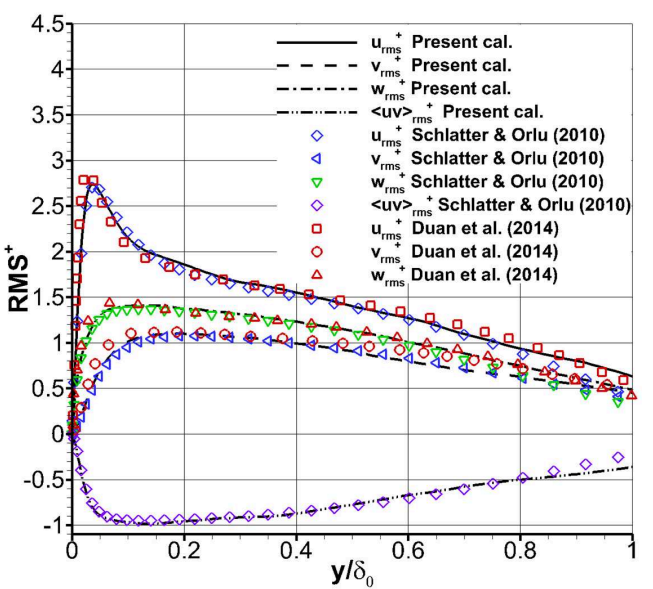

(b) RMS velocity distribution

Figure 3. Mean (a) and RMS (b) velocity distributions obtained at $\mathrm{x}=0 \mathrm{~mm}$ on the flat plate, compared with the incompressible flow data of Schlatter \& Örlü (2010) and supersonic flow data of Duan \& Beekman (2011). The van Driest transformation was applied to the mean velocity and a Morkovin scaling $\xi=\sqrt{\rho / \rho_{w}}$ to the RMS curves.

while the superscript vd represents the value under the van Driest transform. Figure 3 shows that the mean and RMS values agree very well with the DNS results of Schlatter \& Örlü (2010), as found in a recent study on turbulent flow over an expansion corner (Sun et al. 2017). DNS data of supersonic boundary layer from Duan \& Beekman (2011) are also compared in figure 3 and it is found that the current calculation agrees well with the compressible velocity profile and the Reynolds-stress data.

\section{Mean flow and turbulent statistics on concave surfaces}

\subsection{Mean flowfield}

The time averaged density fields together with sonic lines are given in figure 4 for the whole domain on the left and in an enlarged view on the right. It can be seen that the density on the concave surface increases in the main stream and in the boundary layer downstream of the turning point and the compression waves become more evident as the curvature radius decreases. The results correspond to the analysis of Spina et al. (1994), which indicates that the boundary layer on concave walls becomes thinner. The local sonic line is superposed on the contours. Near the outlet, the sonic lines are smooth, with no sign of any artificial disturbances generated near the outflow boundary. An interesting part is that the subsonic region increases as the curvature radius decreases.

Profiles of the time-averaged skin friction, $C_{f}=2 \tau_{w} /\left(\rho_{\infty} U_{\infty}^{2}\right)$, along the wall for the flat plate and the two concave cases are shown in figure 5 . The skin friction starts to follow the expected results (Sun et al. 2017) of a turbulent boundary layer by $x / \delta_{0}=-5$, which indicates that the artificial inflow condition recovers to turbulent boundary layer flow roughly 5 inflow boundary layer thicknesses before the turning point $x / \delta_{0}=0$. The skin friction coefficient decreases slightly in the vicinity of the turning point, and increases further downstream, while the pressure increases consistently downstream of the turning point. Experimental data for the wall pressure distribution in a Mach 2.95 supersonic boundary layer (Wang et al. 2016b) are superposed, showing a reasonable agreement. The deviation cannot be discussed further since the uncertainty is not known from the 

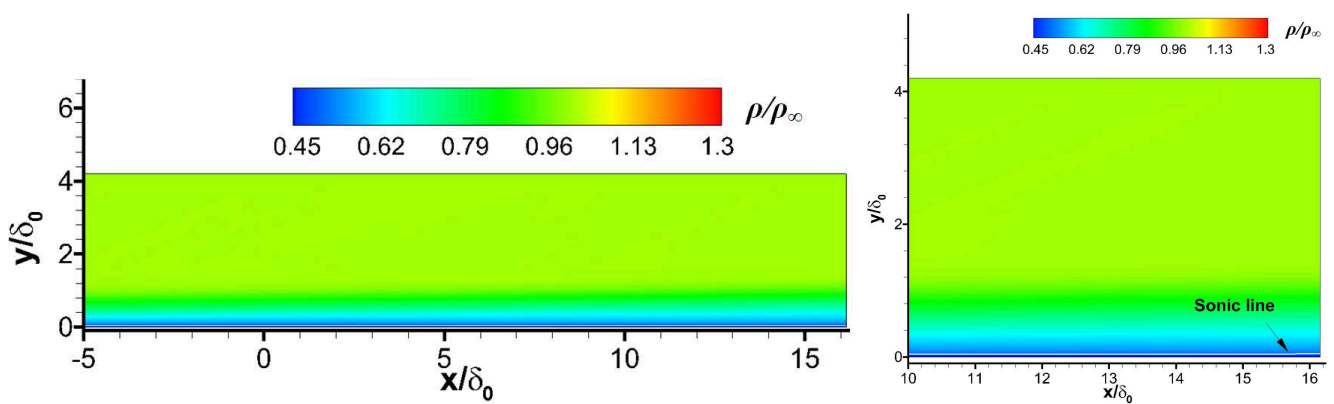

(a) Flat plate
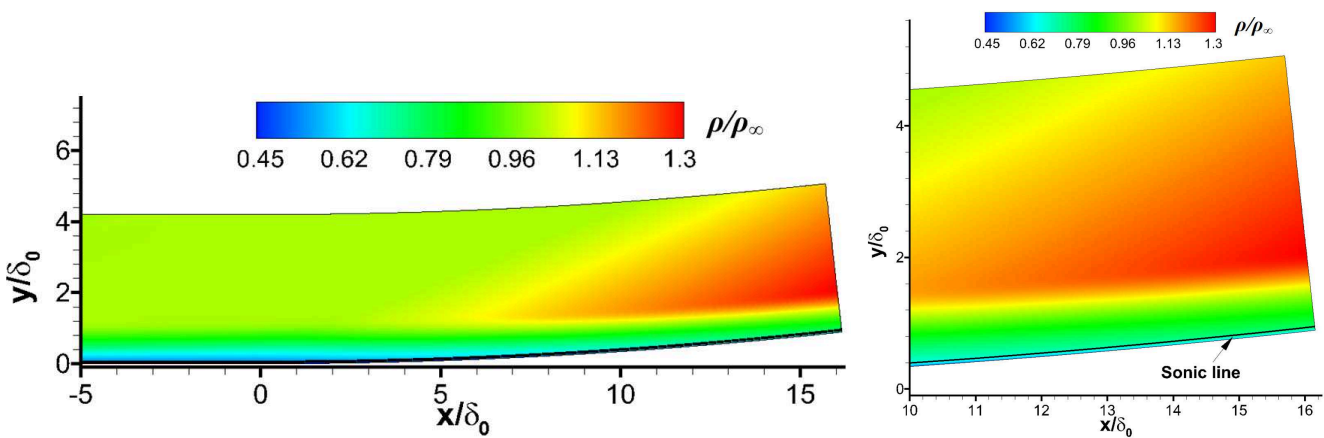

(b) CurvR908mm
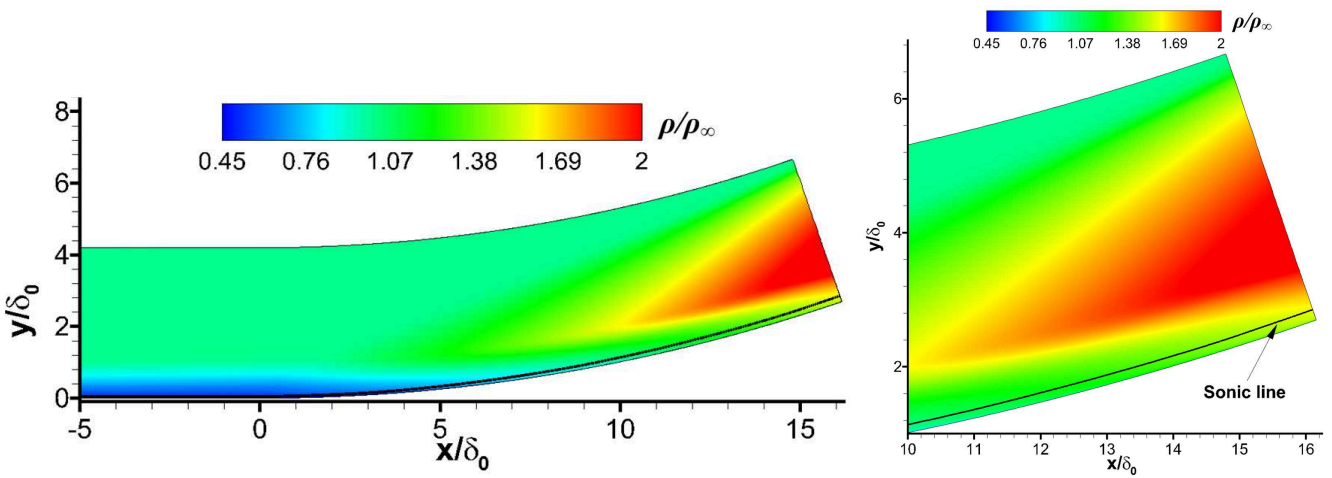

(c) CurvR308mm

Figure 4. Contours of time averaged density of the flat plate and the concave cases, normalized by the inflow freestream density. The local sonic line is superposed on the contour. a) Flat plate, b) CurvR908mm, c) CurvR308mm.

experiments. Figure 5(c) shows the pressure gradient distribution along the wall surface, where it is seen that the pressure gradient has an abrupt increase at $x / \delta_{0}=0.0$ for the concave cases and an approximately linear increase for $x / \delta_{0}>3$. The flow on concave walls experiences a continuously increasing pressure all the way to the outflow.

Figure 6 shows velocity profiles both upstream and downstream of the turning point. It can be seen that for concave cases, the velocity on the concave surface is lower than that on the flat plate over most of the boundary layer. Thus, the downstream velocity profiles become less full as the curvature radius increases. In figure 7 , the velocity profiles are re-plotted again in wall coordinates using the van Driest transformation, which takes into account the variation of density in compressible boundary layers. Compared to the 
10

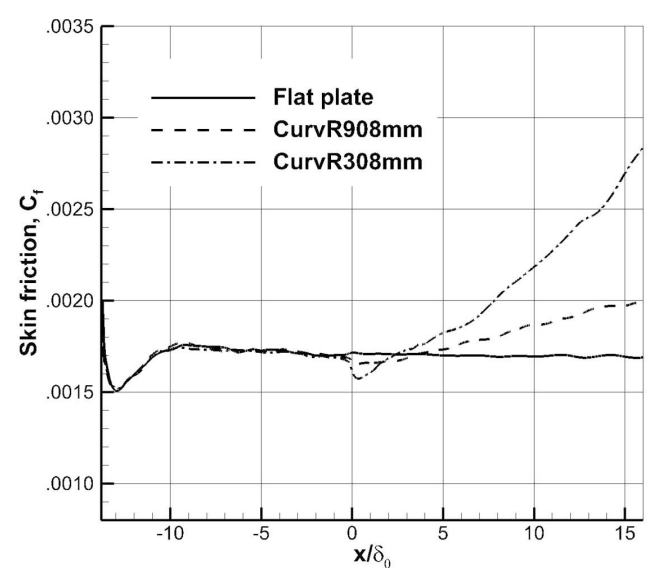

(a) skin friction coefficient

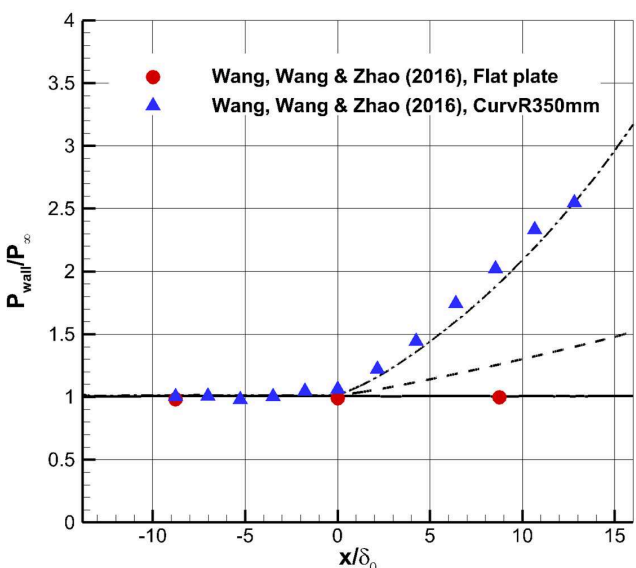

(b) wall pressure normalized by the freestream pressure

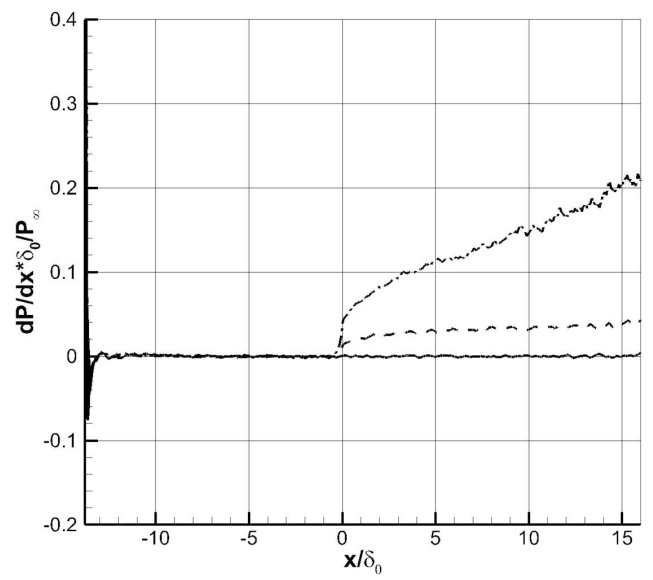

(c) non-dimensional wall pressure gradient

Figure 5. Mean flow parameters along the flat and concave surfaces

velocity profiles of the flat plate case, the velocity profiles on concave surfaces deviate slightly from the log-law around the turning point, and the difference becomes more significant further downstream. In the region near the boundary layer outer edge, the wake layer of the CurvR308mm case deviates significantly from the flat plate case.

The velocity changes differently in the inner part of the boundary layer of concave cases compared to the flat plate case, as shown in figure 8, where the same velocity profiles as given in figure $7(\mathrm{~d})$ are re-plotted using the outer length scale $\delta_{0}$. Under this scaling the velocity near the wall increases, which is opposite to the behaviour in the outer region.

From the turbulent boundary layer velocity profiles, it is possible to analyze the boundary layer displacement thickness $\delta^{*}$ and the momentum thickness $\theta$, which are defined as $\delta^{*}=\int_{0}^{h}\left(1-\frac{\rho}{\rho_{e}} \frac{u}{U_{e}}\right) d y$ and $\theta=\int_{0}^{h} \frac{\rho}{\rho_{e}} \frac{u}{U_{e}}\left(1-\frac{u}{U_{e}}\right) d y$, respectively. Here we integrate up to $h=1.5 \delta_{0}$ to reduce the effects of variation in the freestream properties $\rho_{e}$ and $U_{e}$. Figure $9(\mathrm{a}, \mathrm{b})$ shows the calculated results for different cases. Over the flat plate, both the displacement thickness and momentum thickness grow. Over the concave surfaces, $\delta^{*}$ experiences a drop after the turning point, followed by a rise downstream of $x / \delta_{0} \approx 4.0$ and eventually a reduction. The changes in the higher curvature case are 


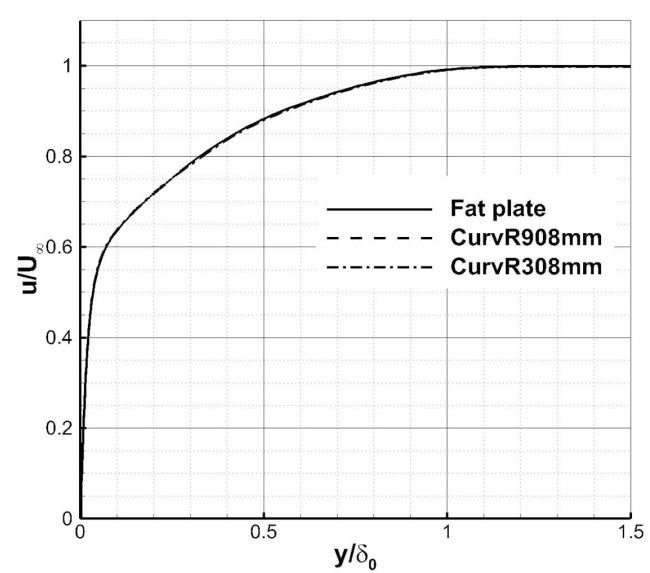

(a)

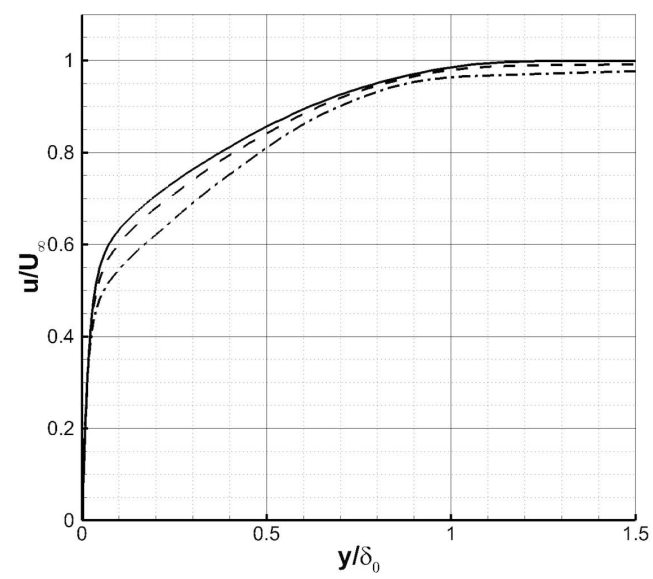

(c)

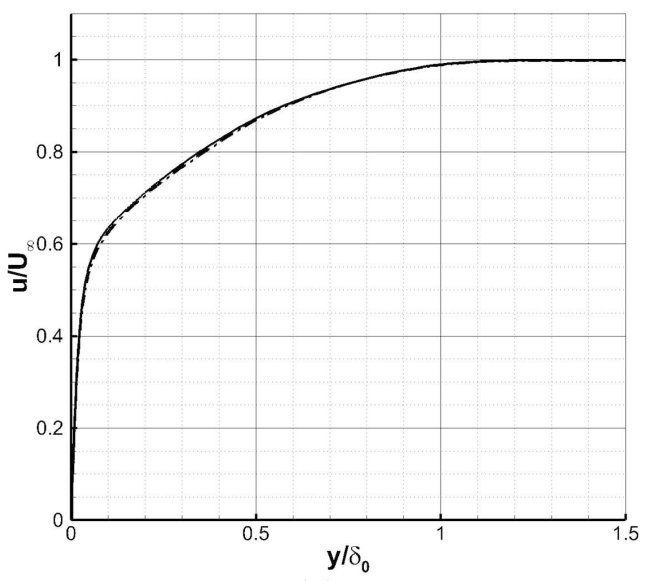

(b)

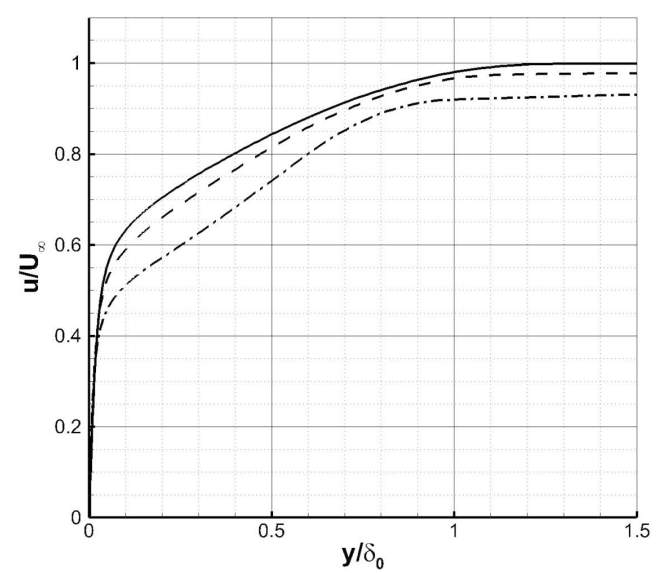

(d)

FiguRE 6. Mean velocity profile predictions at different locations, $(a) x / \delta_{0}=-2.42,(b) x / \delta_{0}=0.81,(c) x / \delta_{0}=5.65,(d) x / \delta_{0}=10.50$, normalized by inflow freestream velocity

more significant. The momentum thickness $\theta$ first rises above the flat plate values after the turning point, then starts to decrease at $x / \delta_{0} \approx 4.0$, with both curved wall cases eventually having lower than the flat plate case.

Figure $9(\mathrm{c})$ shows the shape factor $\left(H=\delta^{*} / \theta\right)$ as a function of the streamwise location of both concave cases. As discussed in previous work (Sun et al. 2017), a higher shape factor represents a velocity profile with less fullness. It is clearly seen that the shape factor increases significantly further downstream of the turning point on the concave surfaces and then reduces. It is found that $\mathrm{H}=8.07$ for CurvR308mm case at $x / \delta_{0}=15.5$, and $\mathrm{H}=5.47$ for CurvR908mm case, compared to $\mathrm{H}=4.83$ for the flat plate case.

Pressure fluctuations were recorded on the wall surface at different positions for 360 non-dimensional time units with 240 samples. The response of the flow to the curvature is examined by comparing the frequency weighted power spectral density(WPSD), which was calculated using a 50\% segmental averaging method as in $\mathrm{Hu}$ et al. (2006). As the flow is homogeneous in the spanwise direction, the WPSD are averaged over the span. The WPSD given in figure 10 show the pressure fluctuations at various locations for different cases. At $x / \delta_{0}=-2.42$ and 0.81 , the pressure fluctuations are almost unchanged 


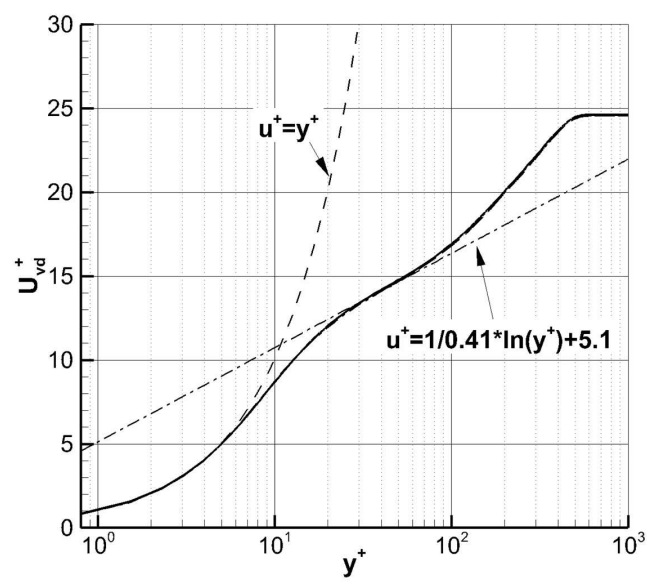

(a)

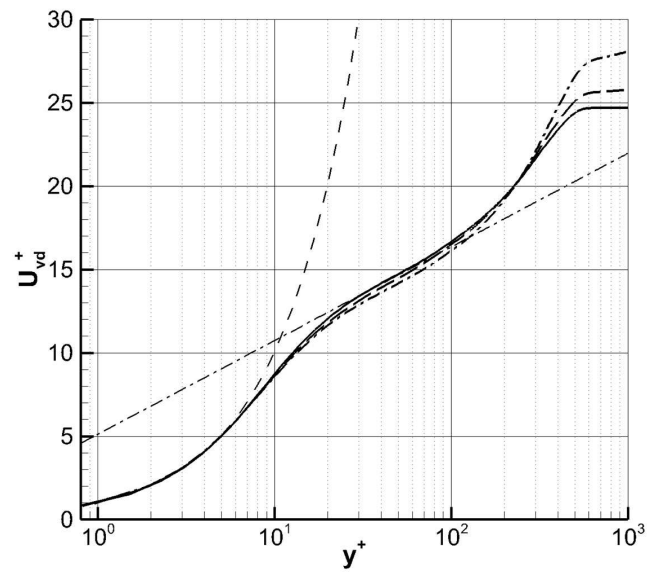

(c)

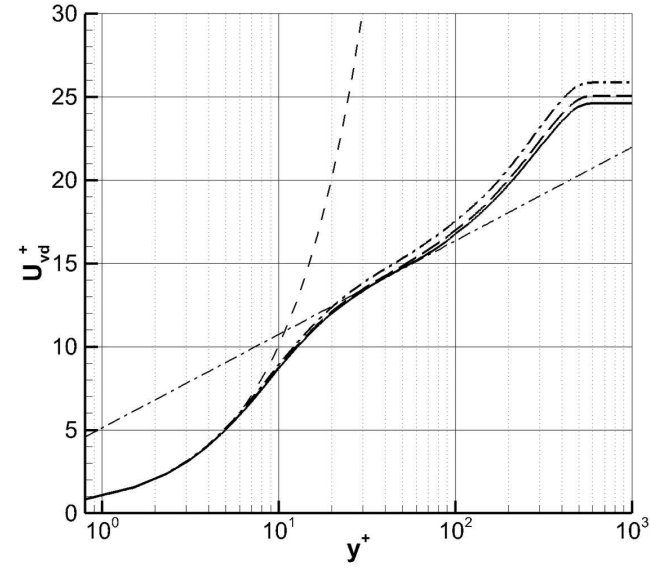

(b)

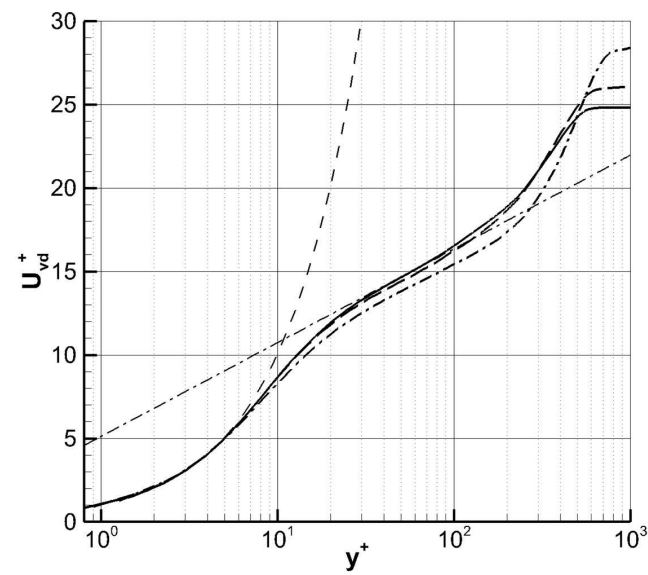

(d)

FiguRE 7. Mean velocity profile predictions at different locations, van Driest transformation is applied to the mean velocity, $(a) x / \delta_{0}=-2.42,(b) x / \delta_{0}=0.81,(c) x / \delta_{0}=5.65,(d) x / \delta_{0}=10.50$

compared to the flat plate case. At $x / \delta_{0}=5.65$, low frequency peaks in the spectra appear for concave cases, while at $x / \delta_{0}=10.50$ the amplitudes are significantly higher throughout the energy-containing range. Thus the magnitude of the turbulent fluctuations increases with the curvature ratio and, in particular, low frequency (presumed to be of large scale) structures appear to be generated on concave walls.

Figure 11 plots RMS values of pressure, temperature and density fluctuation normalized by local averaged $\langle p\rangle,\langle T\rangle$ and $\langle\rho\rangle$. It is seen that the local magnitude of density fluctuations on the concave wall is always larger than on the flat plate. For pressure and temperature fluctuations, the magnitude of the fluctuations increases most obviously in the outer region in the CurvR308mm boundary layer compared to the flat plate. In the inner layer, RMS values of pressure and temperature fluctuations of the CurvR308mm case are lower than the flat plate. Figure 12 shows the plots of RMS values of velocity fluctuation. It is seen that for velocity fluctuations, the three components of the velocity fluctuations are all increased. These quantitative results exhibit the turbulence amplification from different aspects. The velocity and density fluctuations increase over the entire boundary layer but in the inner layer the pressure 


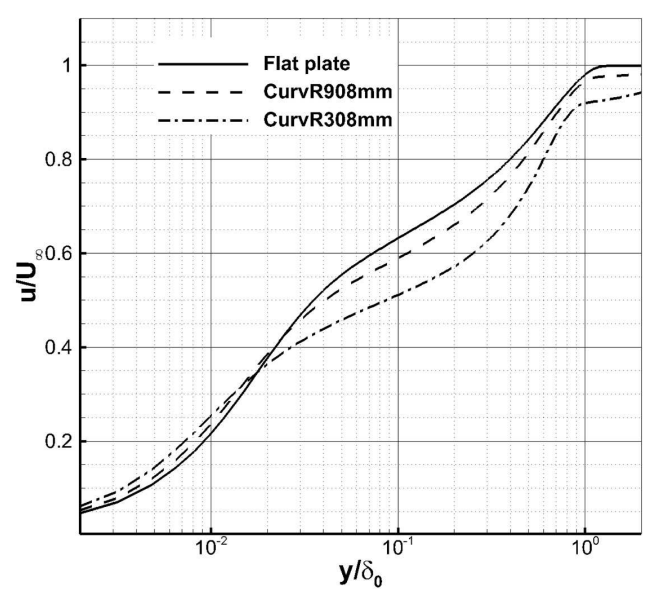

(a)

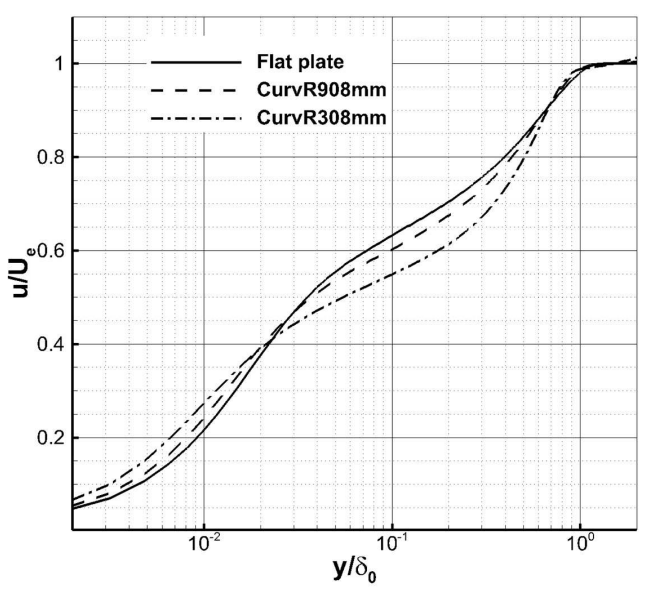

(b)

Figure 8. Distribution of velocity as a function of wall-normal distance at $x / \delta_{0}=10.50$ normalized by inflow freestream velocity $U_{\infty}$ (a) and local freestream velocity $U_{e}$ over the boundary layer (b)

and temperature fluctuations are not enhanced, which might be related to the obvious augmentation of the averaged pressure and temperature due to the concave compression. Recalling figure 2, the comparison of one-dimensional power spectra between the flat plate case and the concave case at $x / \delta_{0}=11.5$ shows that $E_{u_{\alpha} u_{\alpha}}$ of CurvR308mm at $y / \delta_{0}=0.0587$ is larger in the high wave number region compared to the flat plate. At low wave numbers, $E_{u_{\alpha} u_{\alpha}}$ of the CurvR308mm case is comparable to the flat plate, which means that the large scale turbulence is not obviously enhanced in the inner region of the CurvR308mm boundary layer. At $y / \delta_{0}=0.2908, E_{u_{\alpha} u_{\alpha}}$ of CurvR308mm is larger than the flat plate over the whole wave number range. This means that both the small and large scales of turbulence are enhanced.

\subsection{Thermal effects}

The recovery temperature at the wall $T r=281.2 \mathrm{~K}$ is calculated according to $T_{r}=$ $T_{\infty}+r \frac{u_{\infty}^{2}}{2 C_{p}}$, with $r=(\mathrm{Pr})^{1 / 3}$. The recovery temperature has little variation along the curved section. To illustrate the concave wall heat transfer compared to the flat plate, figure 13 gives the Stanton number

$$
S_{t}=\frac{q_{w}}{\rho_{\infty} \rho_{\infty} C_{p}\left(T_{w}-T_{r}\right)}
$$

along the wall, where $q_{w}=-\left.k \frac{d \bar{T}}{d y}\right|_{w}$.

As seen from figure 13, the Stanton number increases on the concave walls compared to the flat plate, and becomes higher as the curvature increases. The temperature profiles shown in figure 14 show that temperature is higher in the outer region of the concave boundary layer compared to the flat plate. The wall-normal temperature gradient for the concave cases decreases in the outer region but increases in the inner region, leading to the observed increase of the Stanton number on the concave wall. For the CurvR308mm case, the temperature decreases more quickly than in the CurvR908mm case and the flat plate. This tendency is similar to the velocity profile along the wall (analyzed in section 3.1), which reflects the different character between the inner layer and the outer layer. The phenomenon is related to the amplification of the turbulence on the concave wall, 
14

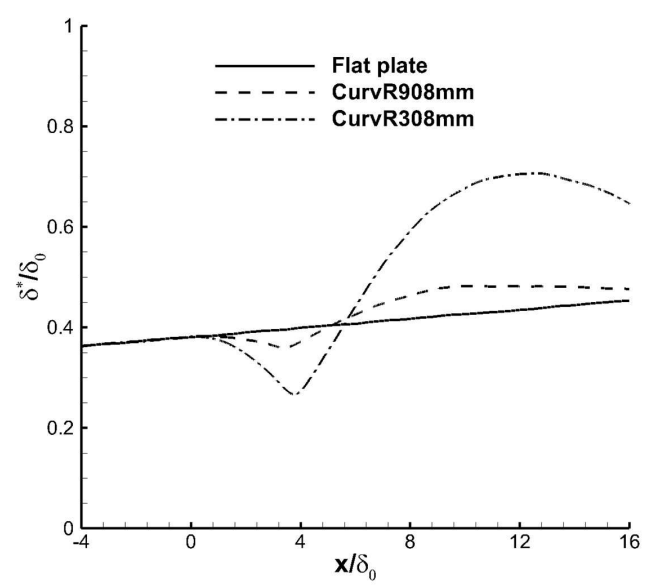

(a)

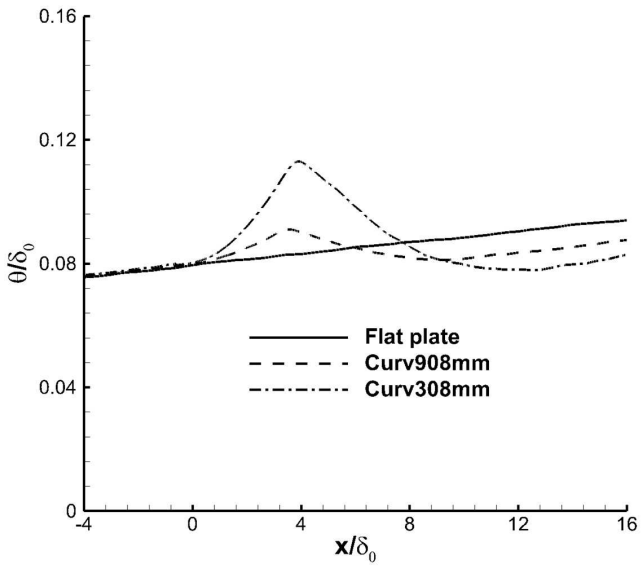

(b)

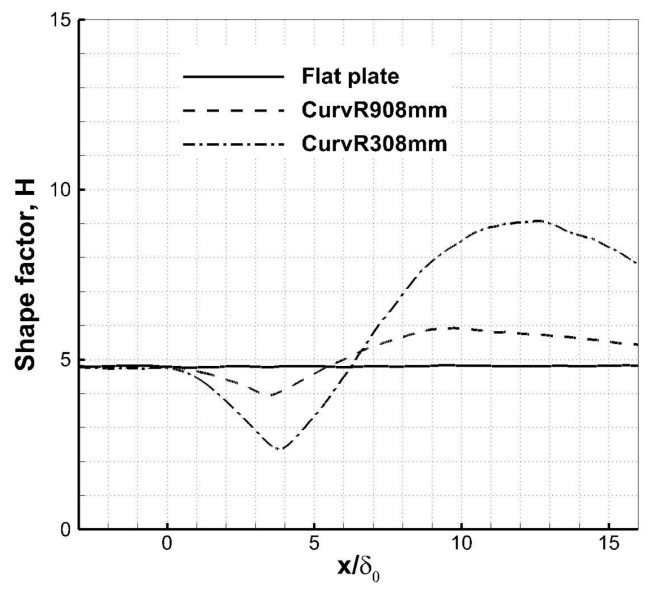

(c)

Figure 9. The displacement thickness $\delta^{*}$ (a) and the momentum thickness $\theta$ (b) and the shape factor $H$ (c) as a function of the streamwise location for the flat and concave cases

and will be analysed in the following sections. A test of the strong Reynolds analogy (SRA) is given in figure 15. Morkovins SRA is well known for compressible turbulent boundary layer flows. The SRA is tested by testing the Morkovin approximate relation (Morkovin 1962)

$$
\frac{\left(\overline{T^{\prime \prime 2}}\right)^{1 / 2} / \tilde{T}}{(\gamma-1) M_{a}^{2}\left(\overline{u^{\prime \prime 2}}\right)^{1 / 2} / \tilde{u}} \approx 1
$$

as well as considering the correlation of streamwise velocity and temperature, according to

$$
R_{u^{\prime \prime} T^{\prime \prime}}=\frac{-\overline{u^{\prime \prime} T^{\prime \prime}}}{\left(\overline{u^{\prime \prime 2}}\right)^{1 / 2}\left(\overline{T^{\prime \prime 2}}\right)^{1 / 2}}
$$

where a tilde denotes Favre average. Figure 15 shows the results at different streamwise locations. Note that the reference boundary layer thickness $\delta_{0}=6.19$ is measured at 


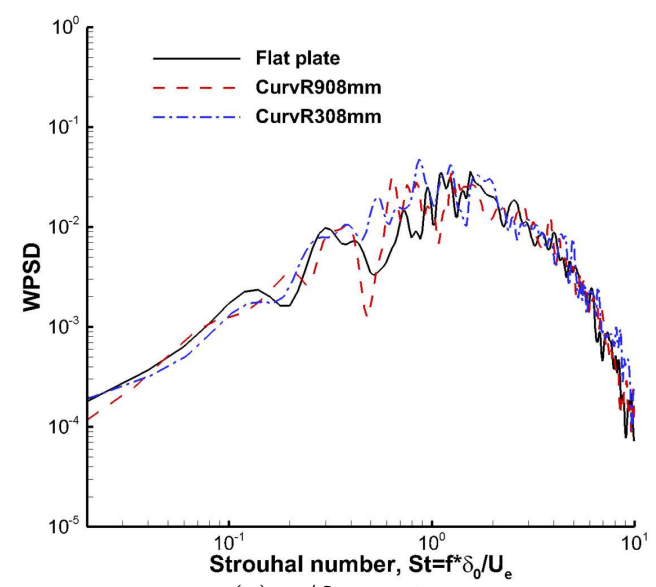

(a) $x / \delta_{0}=-2.42$

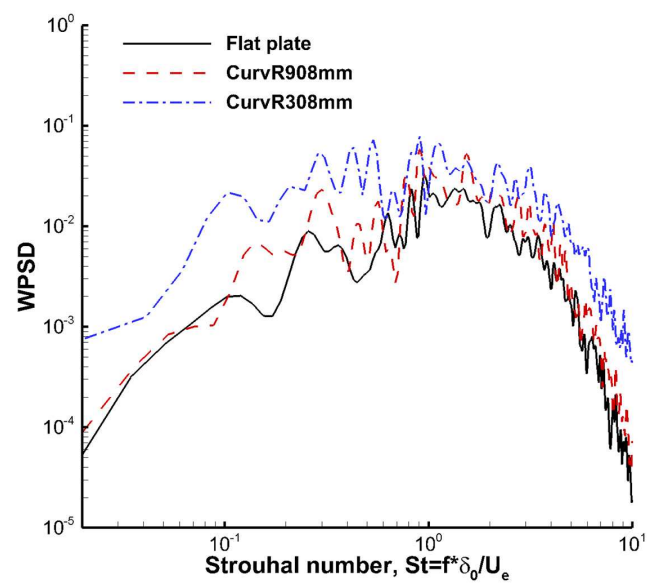

(c) $x / \delta_{0}=5.65$

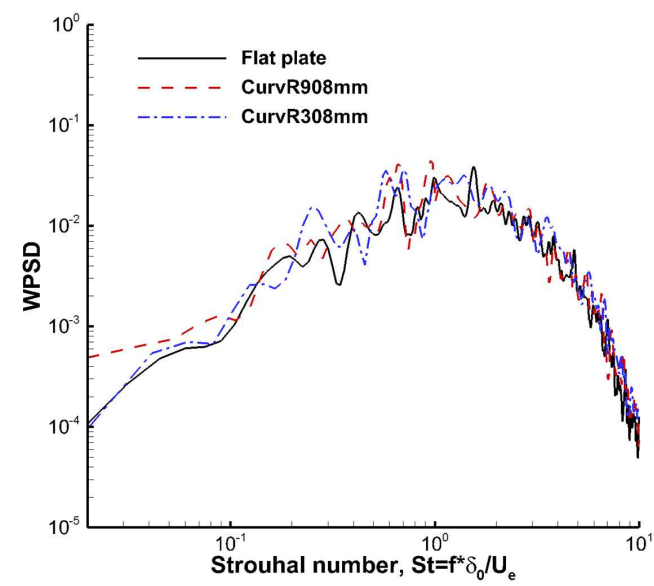

(b) $x / \delta_{0}=0.81$

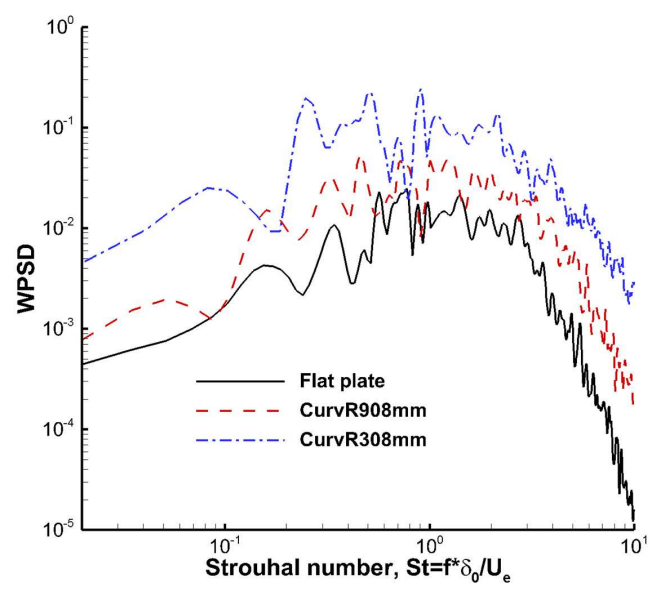

(d) $x / \delta_{0}=10.5$

FigURE 10. Weighted power spectra density (WPSD) of pressure on the flat plate and concave walls, normalized by square of freestream dynamic pressure
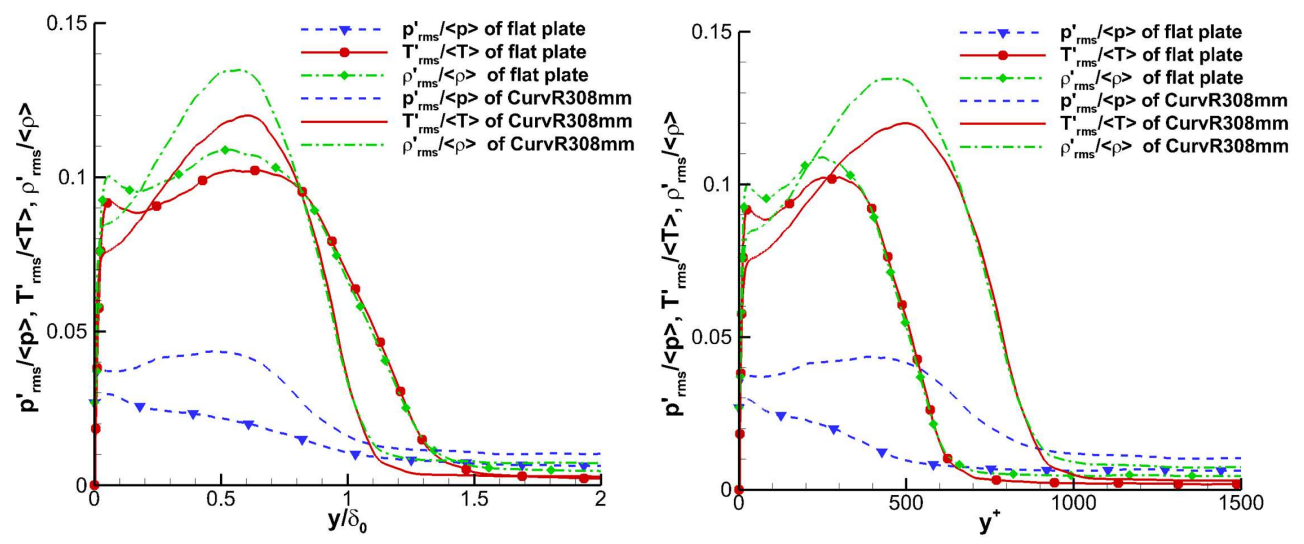

FiguRE 11. $p^{\prime}{ }_{r m s} /<p>, \rho^{\prime}{ }_{r m s} /<\rho>$ and $T^{\prime}{ }_{r m s} /<T>$ versus $y / \delta_{0}$ (left) and $y$ unit (right) for different cases at $x / \delta_{0}=11.5$ 

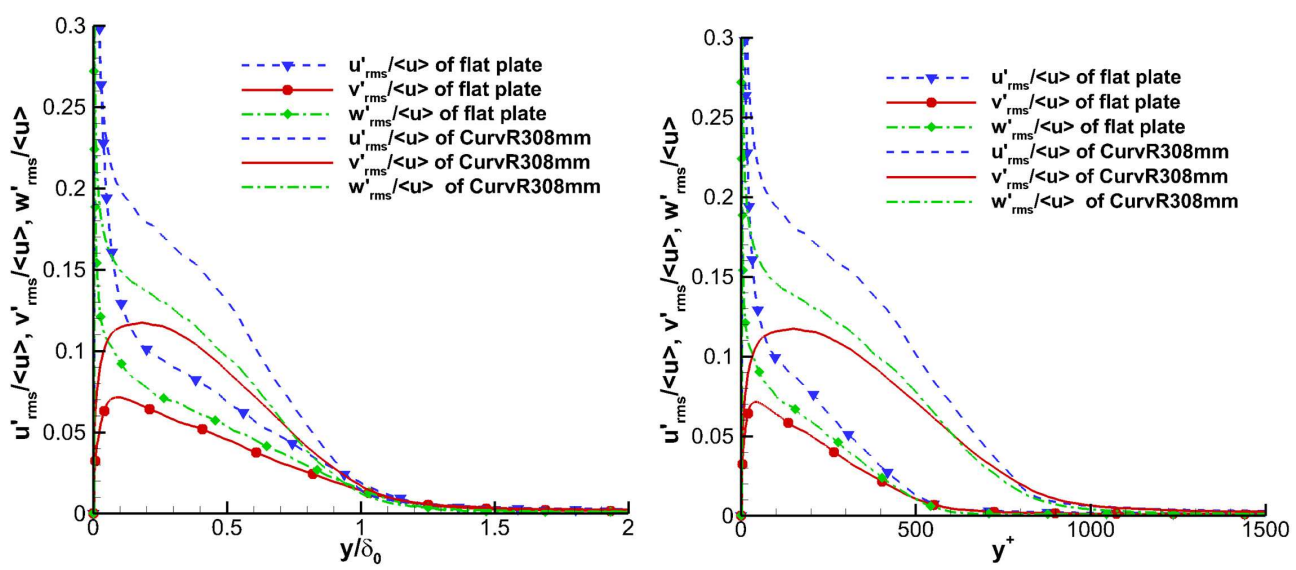

Figure 12. Turbulence intensities of the fluctuating velocity components versus $y / \delta_{0}$ (left) and $y$ unit (right) for different cases at $x / \delta_{0}=11.5$

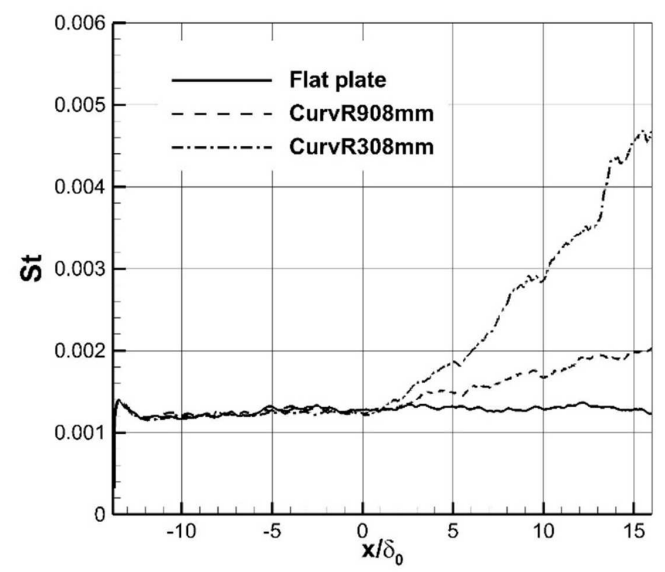

Figure 13. Stanton Number along the wall in the streamwise direction

the turning point $x / \delta_{0}=0.0$, and the boundary layer on the concave wall is thinner than on the flat plate.That is why figure 15(b) seems slightly different at the edge of the boundary layer.The results show that the SRA relations are satisfied and $R_{u^{\prime \prime} T^{\prime \prime}}$ is almost independent of wall temperature except close to the wall.Through most of the boundary layer, $R_{u^{\prime \prime} T^{\prime \prime}}$ is approximately 0.6 , similar to the results reported by Guarini et al. (2000), Maeder et al. (1998), Pirozzoli et al. (2004) and Duan \& Beekman (2011). On the concave wall, the SRA relations tend to be satisfied slightly better than along the flat plate. 


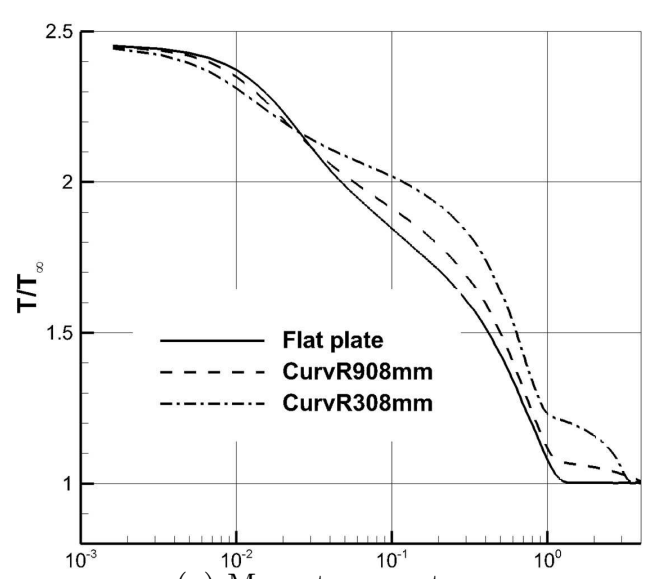

(a) Mean temperature

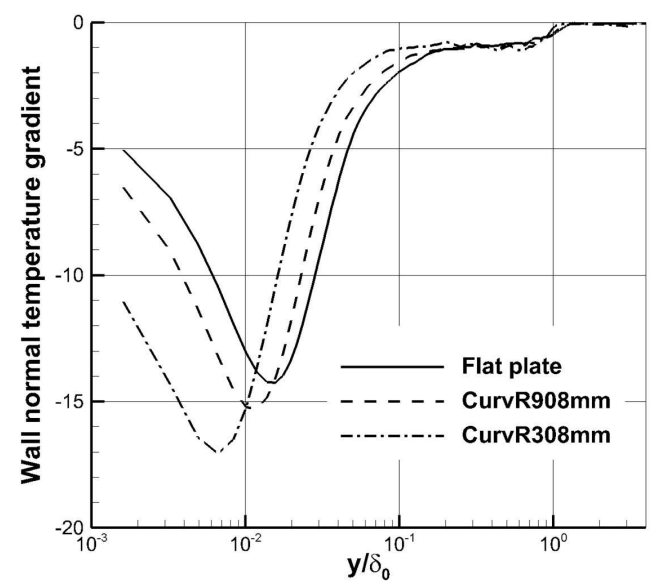

(b) wall-normal temperature gradient

Figure 14. Mean temperature and wall-normal gradient profiles at $x / \delta_{0}=10.50$

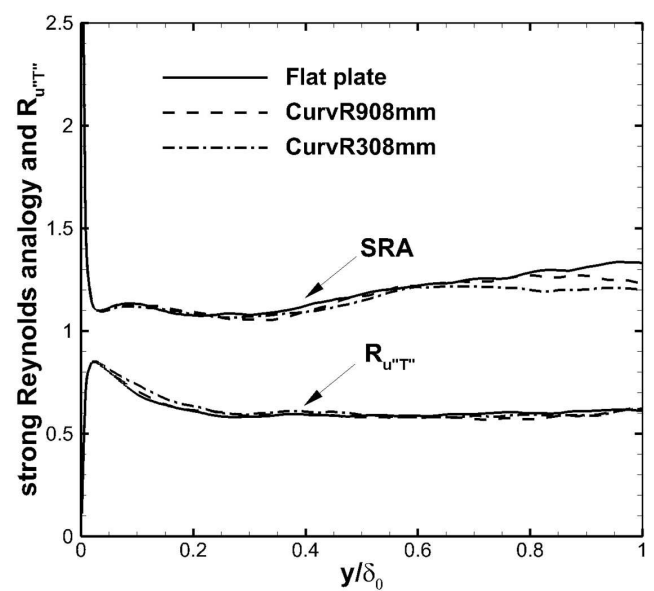

(a) $x / \delta_{0}=0.81$

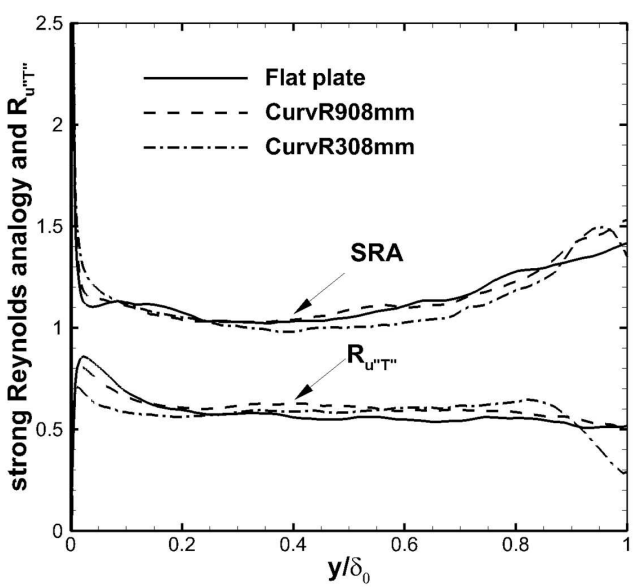

(b) $x / \delta_{0}=10.50$

FiguRE 15. SRA profiles at (a) $x / \delta_{0}=0.81$ and (b) $x / \delta_{0}=10.50$

\section{Instantaneous turbulent structures in the concave flowfield}

\subsection{Turbulence flow structures}

Contours in a 2D slice through instantaneous density fields are given in figure 16 . It can be seen that the density on the concave surface increases in the main stream and in the boundary layer downstream of the turning point, and also that compression waves become evident as curvature radius decreases. As measured in previous studies(Fernando \& Smits (2006), Donovan et al. (1994), Spina et al. (1994), Flaherty \& Austin (2013a), Wang et al. $(2016 b))$, the boundary layer thickness decreases on a concave wall and on a flat plate with adverse pressure gradient in a supersonic flow. In contrast to this, recent by nano-particle planar laser scattering visualizations of the instantaneous two-dimensional flow Wang \& Wang (2016) observed that the concave boundary layer becomes thicker. From the velocity profiles in the previous section we see that in the present study the boundary layer is on average thinner on concave walls, as seen also in the instantaneous slice shown on figure 16c. However, another slice at a different spanwise location (figure 

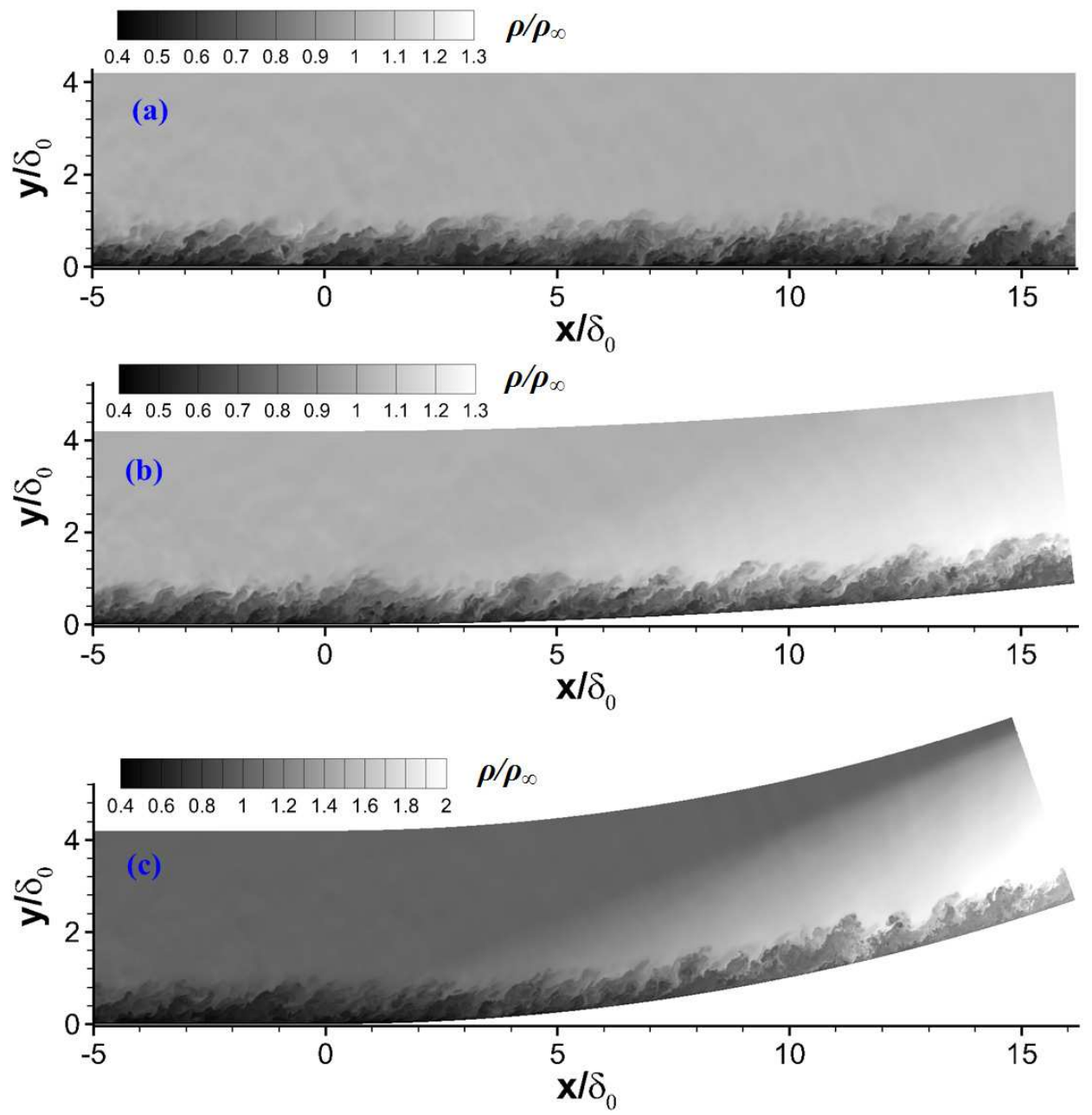

Figure 16. Contours of density flowfields of the flat plate and the concave cases at $z / \delta_{0}=1.2$, a) flat plate, b) CurvR908mm, c) CurvR308mm, normalized by the inflow freestream density

17) shows large structures that might be identified as an instantaneous increase of the boundary layer thickness, which might explain the results of Wang \& Wang (2016). This will be discussed further in section 4.3 and we attribute the variability in thickness to the formation of destabilizing structures with strong instantaneous spanwise variability.

In order to investigate vortex structures before and after the turning point, figure 18 shows an iso-surface of $\lambda_{2}$, which is the second eigenvalue of the $3 \times 3$ matrix comprising the velocity gradient components Jeong \& Hussain (1995). A small negative value $\left(\lambda_{2}=-0.3\right)$ is selected to visualize the turbulence structures. Compared with the flat plate, supersonic flow past concave surfaces shows an augmentation of $\lambda_{2}$ vortices. The turbulence is rapidly amplified in the CurvR308mm case, as shown in figure 18(c). Recalling figure 16(c) and figure 17, a careful observation of the turbulent structures on the concave wall demonstrates that many smaller fluctuations are superimposed on the large scale structures. Moreover it is found that new large scale structures form in the downstream boundary layer on the curved surface. 


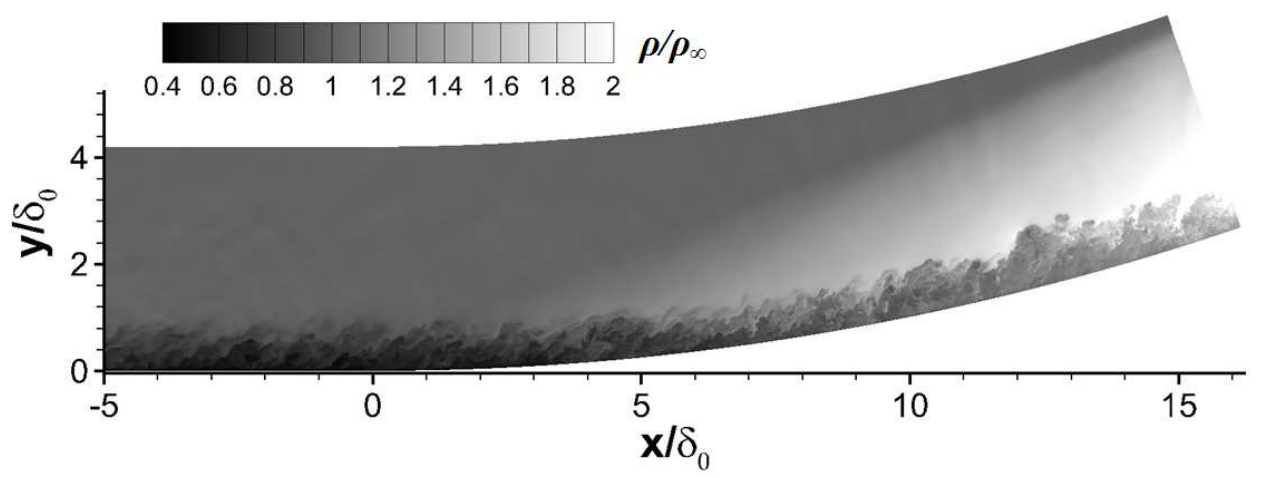

FIGURE 17. Contours of density flowfields of CurvR308mm case at $z / \delta_{0}=1.8$, normalized by the inflow freestream density

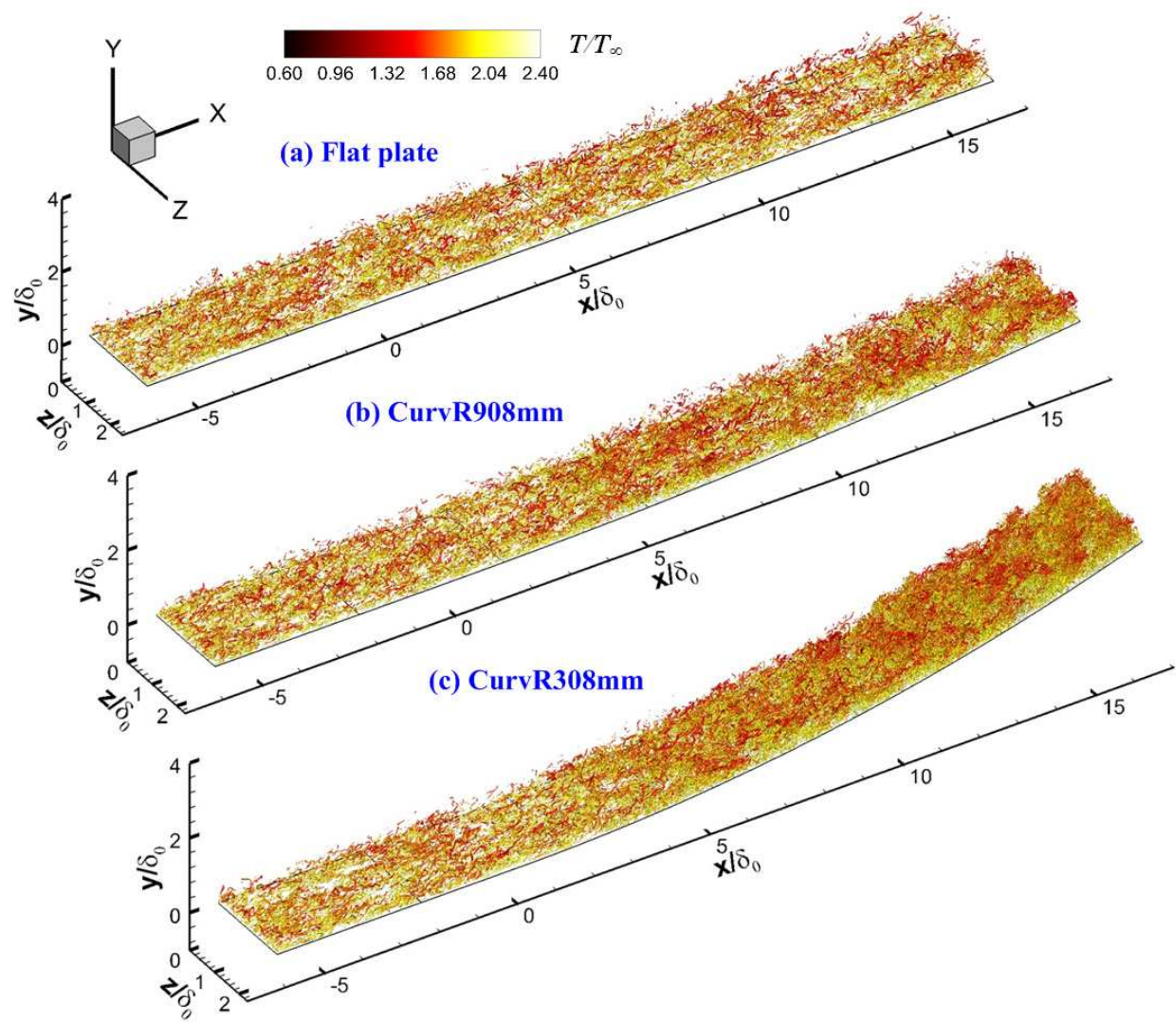

FIGURE 18. Iso-surface of $\lambda_{2}$ coloured by the instantaneous temperature of different cases, a) flat plate, b) CurvR908mm, c) CurvR308mm

\subsection{Comparison of near-wall streaks and spanwise two-point correlations}

The near-wall streaks in the turbulent flowfield of CurvR308mm case are analyzed in this section. For the benefit of comparisons between concave cases and the flat plate, the concave wall is plotted in a body-fitted coordinate system $(\xi, \eta)$, transforming the computational domain to a rectangular box. The transformed velocity components are 


\section{\begin{tabular}{l|l|ll}
\hline & & & $\boldsymbol{u} t$
\end{tabular} $\boldsymbol{U}_{\infty}$ \\ $\begin{array}{llll}0.1 & 0.2 & 0.3 & 0.4\end{array}$}

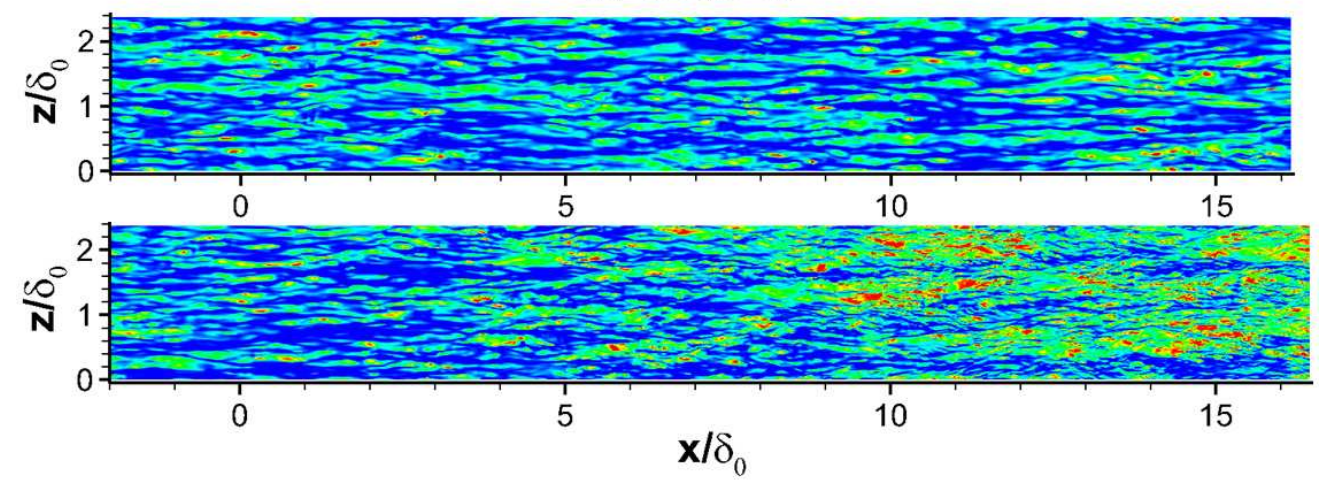

(a) at $y / \delta_{0}=0.0068$ for the flat plate (top) and the CurvR308mm (bottom) cases
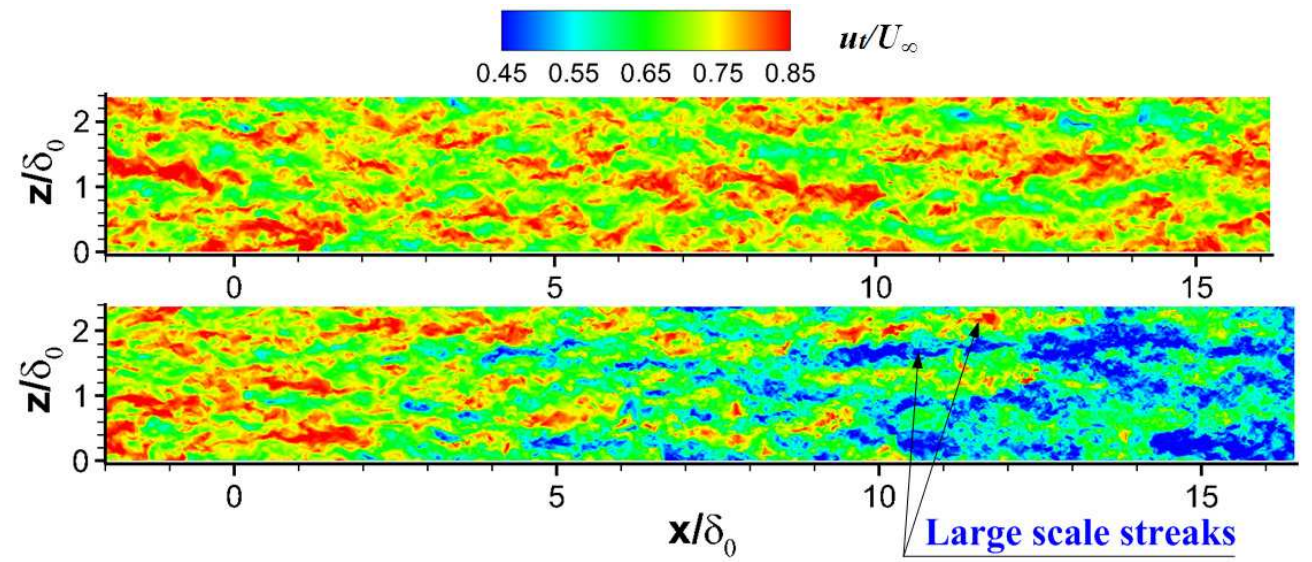

(b) at $y / \delta_{0}=0.255$ for the flat plate (top) and the CurvR308mm (bottom) cases

Figure 19. Streamwise velocity contours at (a) $y / \delta_{0}=0.0068$ and (b) $y / \delta_{0}=0.255$ slices of the flat plate (top) and the CurvR308mm (bottom) cases

given by $u_{t}=u \cos \beta-v \sin \beta$ and $v_{t}=u \sin \beta+v \cos \beta$ respectively, where $\beta$ is the local wall turning angle (see Figure 1).

The streamwise velocity fields in the transformed coordinates at the same wall-normal distance $\left(y / \delta_{0}=0.0068\right)$ are shown in figure $19(\mathrm{a})$ from a top view. Alternating low and high speed streaks can be clearly identified. The classic near-wall streaks occur in the upstream undisturbed boundary layer in both cases. In the inner layer further downstream, however, the quasi-streamwise structures break down into smaller vortices and an abundance of small structures are generated along the concave wall. The distance between neighbouring low-speed streaks decreases on the concave wall compared to the flat plate case, which will be quantified in the next paragraph with the two-point correlation results. At $y / \delta_{0}=0.255$, shown on figure $19(\mathrm{~b})$, the minimum streamwise velocity of the concave case is lower than the flat plate case at the same location, as discussed earlier. Large scale streaks with enlarged streak spacing are seen on the concave wall, and are found to remain coherent over a long streamwise distance.

To quantify the distance between two neighboring low-speed streaks, two-point spanwise correlations of velocity perturbations are compared between the flat plate and the 


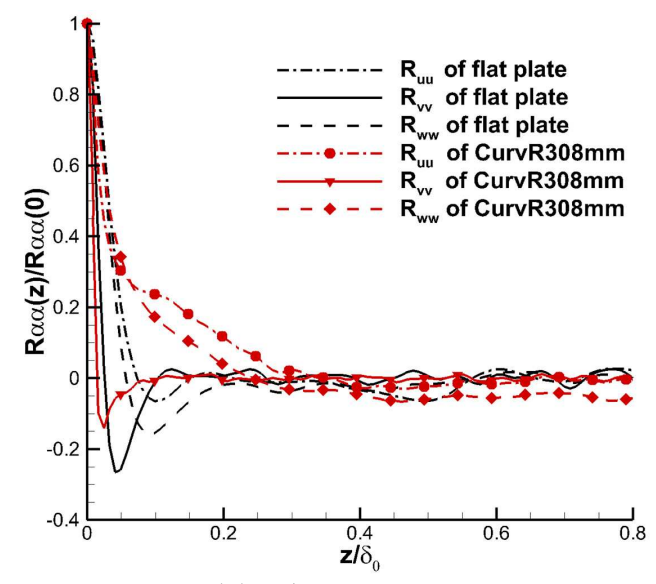

(a) $y / \delta_{0}=0.0068$

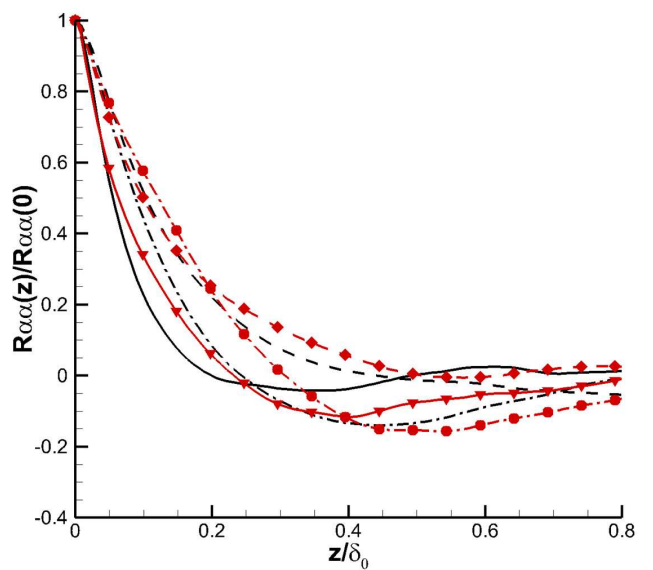

(b) $y / \delta_{0}=0.255$

FiguRE 20. Two-point spanwise correlations for velocity perturbations at $x / \delta_{0}=15.5$. The spanwise coordinates are normalized by the boundary layer thickness $\delta_{0}$.

CurvR308mm cases at $x / \delta_{0}=15.5$ for different wall-normal distance, as given in figure 20. At $y / \delta_{0}=0.0068$ (figure 20a) in the flat plate case, the minimum of the wall-normal velocity correlation is found at $z / \delta_{0}=0.045$, which is the typical streak spacing in a zero-pressure gradient boundary layer. For the CurvR308mm case, the streak width is significantly reduced, consistent with the increasing skin friction. This is in contrast to the behaviour away from the wall, where at $y / \delta_{0}=0.255$ (figure 19), an increase in the streak spacing compared to the flat plate case can be identified. This indicates that streaks might be organized into new patterns, corresponding to the large velocity streaks seen in figure 19.

\subsection{Iso-surfaces and slices of instantaneous velocity in three-dimensional flowfield}

To understand the changes in coherent structures, iso-surfaces of the streamwise velocity, together with streamlines coloured by the wall-normal distance are illustrated and compared in figure 21-22. All the results are based on the domain transformation introduced in Section 4.2.

From figure 21, it is clear that smaller concave curvature radius (CurvR308mm), which means higher curvature ratio, tends to destabilize structures in the boundary layer. For the flat plate case shown in figure 21(a), we can clearly see the classic elongated streamwise structures in the iso-surface of $u_{t}=0.4$. For the CurvR $908 \mathrm{~mm}$ case, the streaks are preserved, but the streaks appear more corrugated and lifted away from the concave wall. Upstream and for a short distance downstream $\left(x / \delta_{0}<5\right)$ of the turning point, the structures are very similar to the flat plate case. However, further downstream the structures start to produce smaller streaks. Most of the structures remain stay close to the wall as in the flat plate case. For CurvR308mm case the streaks on the concave wall develop into a new pattern and large scale organized structures are lifted away from the wall into the outer layer. The orientation of these structures suggests that they are likely to be the Görtler-like structures previously shown in numerical simulations of hypersonic flow(Ren \& Fu 2015a). From a comparison of figures 21(a)-(c), we can see that the concave effects strengthen the liftup process of velocity streaks in the boundary layer, with the Görtler-like vortices pumping fluid packets from the inner layer outwards. Recalling the pressure fluctuation discussed in Section 3.2, since Görtler instabilities 


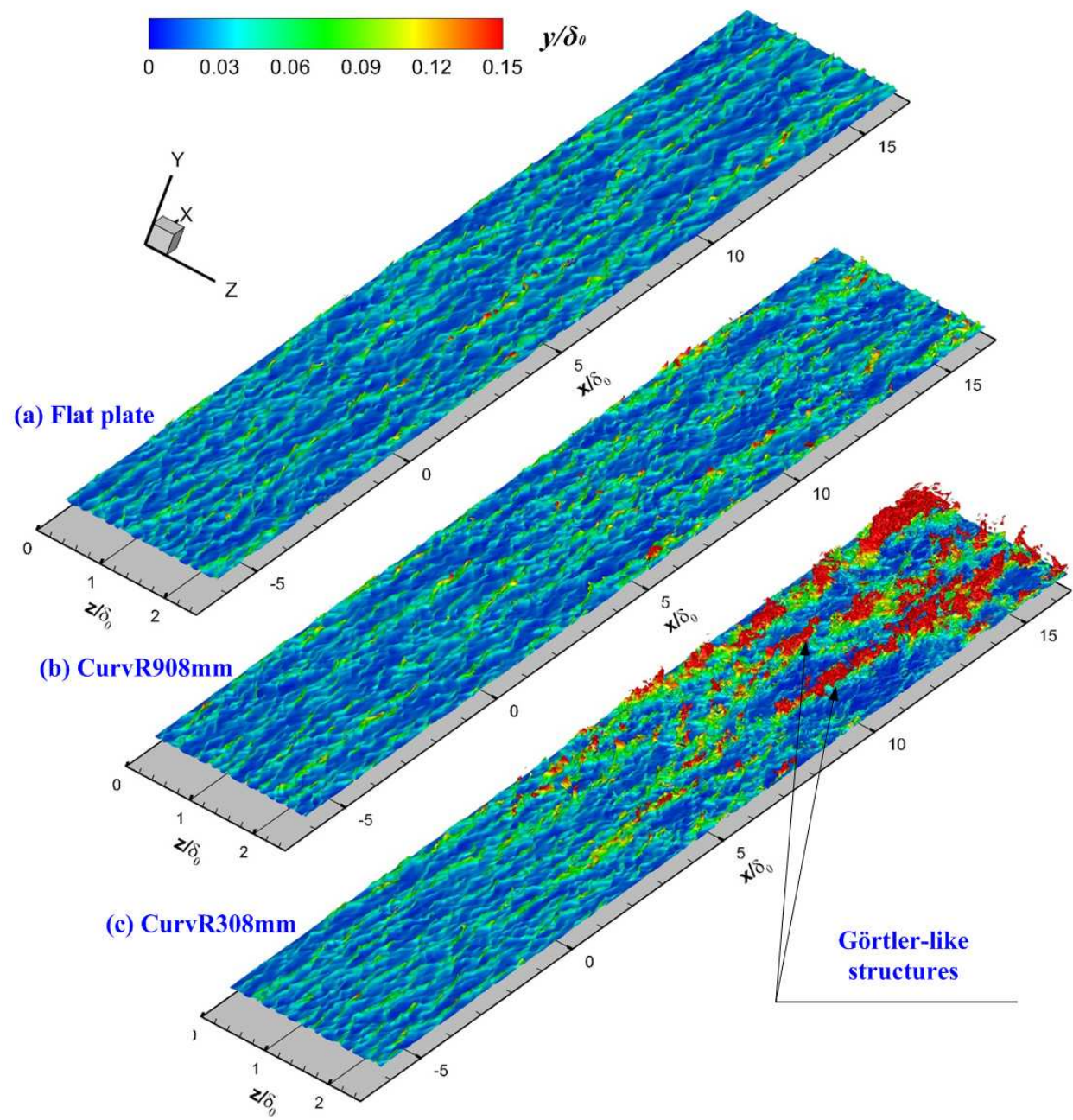

FiguRE 21. Turbulence coherent structures visualized with the iso-surface of $u_{t} / U_{\infty}=0.4$ coloured by the wall-normal distance, (a) Flat plate, (b) CurvR908mm, (c) CurvR308mm

lead to coherent large scale structures in the concave boundary layer, the reason for the low frequency pressure fluctuations becomes apparent. Observation on the iso-surface of $u_{t}=0.4$ at different time points reveals that the Görtler-like structures drift in the spanwise direction as time changes. Since they are not fixed in space, these structures cannot be investigated from an averaged view.

Figure 22 shows representative instantaneous streamlines, originating in the $y / \delta_{0}=0.0266$ plane and $x / \delta_{0}=-6.0$ upstream of the turning point. These streamlines, coloured by the wall-normal distance, illustrate the motion of the fluid. It is seen that the initially planar streamlines are lifted and tilted downstream of the turning point. For the flat plate case, the streamlines concentrate into streaky structures and are convected downstream. For the concave cases, the streamlines form into lifted streaks, consistent with the generation of Görtler-like vortices, and illustrate how the streamlines transport fluid from the upstream inner boundary layer to the outer layer downstream.

Figure 23(a) shows contours of the local velocity contour superimposed with the local 


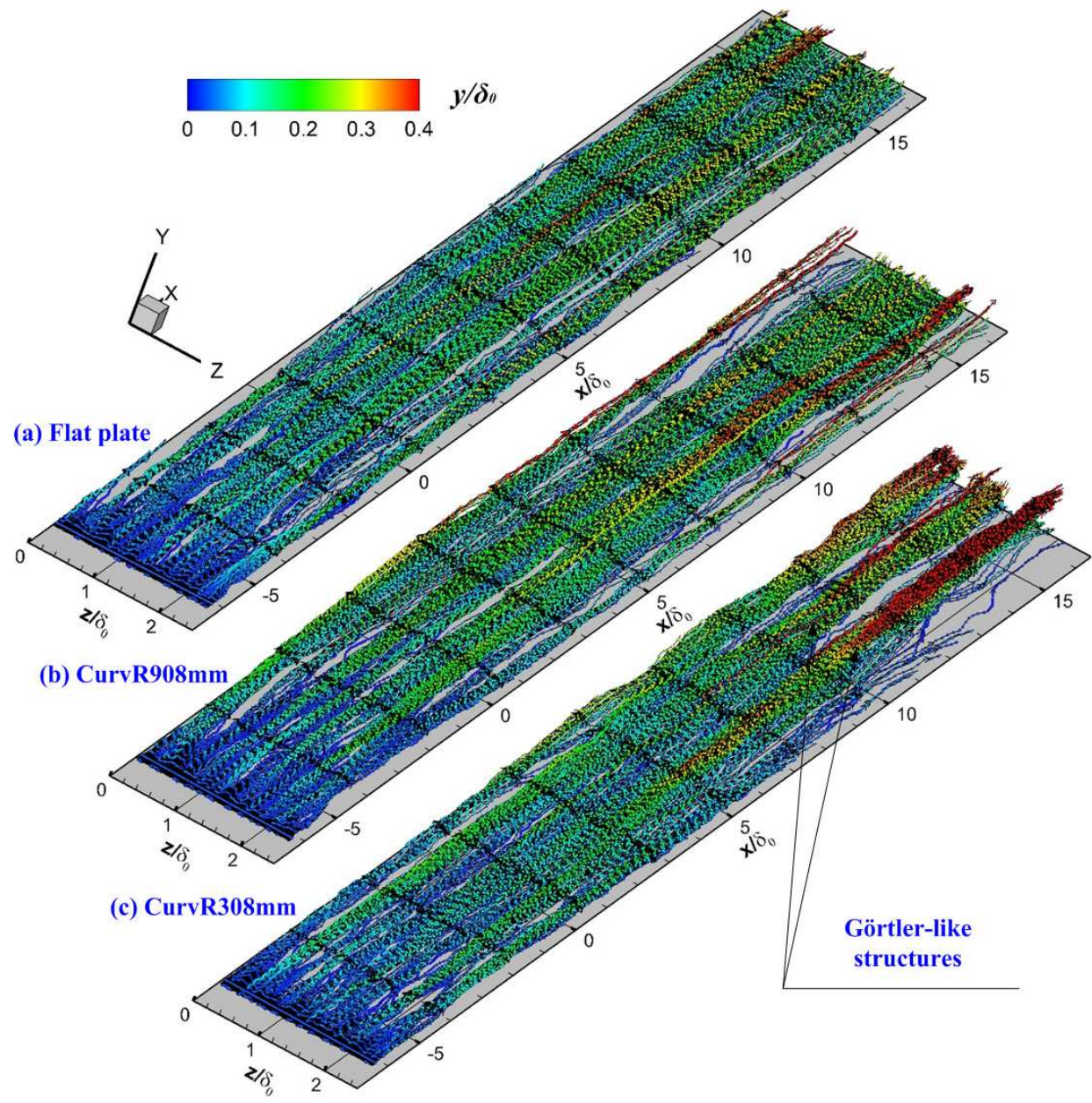

FIGURE 22. Oblique view of three-dimensional streamlines originating from inflow wall parallel plane $y / \delta_{0}=0.0266$ and $x / \delta_{0}=-6.0$, colored by the wall-normal distance in $y / \delta_{0}$ unit, a) Flat plate, b) CurvR908mm, c) CurvR308mm

in-plane streamlines for the instantaneous flow at $x / \delta_{0}=11.5$. The predominant wallnormal flow on concave surface outside boundary layer is due to surface curvature centrifugal effect. The region shown in the dashed-line window is an example of a mushroom structure, which is likely to be the typical consequence of a Görtler vortex, as in concave laminar flow (Ren \& Fu 2015a). It is seen that the low-momentum fluid in the inner boundary layer is pumped into the outer layer by the Görtler-like structures.Figure 23(b) shows that small scale vortices are more abundant on the concave wall. Combined with the $\lambda_{2}$ vortex structures in figure 18(c) and the streaks in the inner boundary layer in figure 19, we can tell that small-scale turbulence is enhanced in the concave boundary layer. The vorticity transport equation can be written as,

$$
\frac{D \omega}{D t}=(\omega \cdot \nabla) \mathbf{u}-\omega(\nabla \cdot \mathbf{u})-\frac{\nabla p \times \nabla \rho}{\rho^{2}}+\nabla \times\left(\frac{\nabla \cdot \tau}{\rho}\right)
$$

The third term, $\frac{\nabla p \times \nabla \rho}{\rho^{2}}$ describes vorticity production via the baroclinic mechanism, which occurs when the density and pressure gradients are misaligned. Pressure enters the 
vorticity equation only through this baroclinic term. It is important to note that vorticity can be generated by baroclinic torque in the concave flow, whereas the other terms in the vorticity equation describe the amplification, stretching, bending or diffusion of existing vorticity. The baroclinic production term is given by

$$
\begin{aligned}
\mathbf{B}_{p}= & \frac{(\nabla p \times \nabla \rho)}{\rho^{2}}=\frac{1}{\rho^{2}}\left(\frac{\partial \rho}{\partial y} \frac{\partial P}{\partial z}-\frac{\partial \rho}{\partial z} \frac{\partial P}{\partial y}\right) \vec{i}+\frac{1}{\rho^{2}}\left(\frac{\partial \rho}{\partial z} \frac{\partial P}{\partial x}-\frac{\partial \rho}{\partial x} \frac{\partial P}{\partial z}\right) \vec{j} \\
& +\frac{1}{\rho^{2}}\left(\frac{\partial \rho}{\partial x} \frac{\partial P}{\partial y}-\frac{\partial \rho}{\partial y} \frac{\partial P}{\partial x}\right) \vec{k}
\end{aligned}
$$

or

$$
\begin{aligned}
\frac{(\nabla p \times \nabla \rho)}{\rho^{2}}= & \frac{1}{\rho^{2}} r_{\eta} r_{\zeta}\left(\frac{\partial \rho}{\partial \eta} \frac{\partial P}{\partial \zeta}-\frac{\partial \rho}{\partial \zeta} \frac{\partial P}{\partial \eta}\right) \vec{i} \\
& +\frac{1}{\rho^{2}} r_{\xi} r_{\zeta}\left(\frac{\partial \rho}{\partial \zeta} \frac{\partial P}{\partial \xi}-\frac{\partial \rho}{\partial \xi} \frac{\partial P}{\partial \zeta}\right) \vec{j}+\frac{1}{\rho^{2}} r_{\xi} r_{\eta}\left(\frac{\partial \rho}{\partial \xi} \frac{\partial P}{\partial \eta}-\frac{\partial \rho}{\partial \eta} \frac{\partial P}{\partial \xi}\right) \vec{k}
\end{aligned}
$$

which indicates the rate at which the vorticity is generated.

Figure 23(c) shows the distribution of the baroclinic production term on a slice at $x / \delta_{0}=11.5$. The baroclinic production term in the outer region of the concave boundary layer has a higher magnitude than on the flat plate and arises near the interface of the high speed flow with the low speed flow, corresponding to the Görtler-like structures shown in figure 23(a). In Equation (4.2), the normal pressure gradient $\partial P / \partial y$ (from centrifugal effects) can interact with spanwise density gradient $\partial \rho / \partial z$, leading to streamwise vorticity induced by these interactions. Therefore it is concluded that the Görtler-like vortices occurred in the outer region of the concave boundary layer can easily twist the original density and pressure gradients. Prominent pressure gradients interact with the density gradients and induce significant vorticity in the concave boundary layer. This corresponds to the generation of the abundant small vortices shown in figure 23(b). In the inner region, pressure gradients interact with the local density gradients and cause more vortices than the flat plate with zero pressure gradient while the influence of the Görtler instability on density gradients appears smaller.

The evolution of turbulent flow structures can be quantified using the anisotropy invariant map (Lumley 1978). This map comprises the second and third invariants of the Reynolds stress anisotropy tensor $\left(b_{i j}\right)$, which are defined as,

$$
\begin{aligned}
& b_{i j}=\frac{\left\langle\rho u_{i}^{\prime \prime} u_{j}^{\prime \prime}\right\rangle}{\left\langle\rho u_{k}^{\prime \prime} u_{k}^{\prime \prime}\right\rangle}-\frac{1}{3} \delta_{i j} \\
& I I_{b}=b_{i j} b_{j i} \\
& I I I_{b}=b_{i j} b_{j k} b_{k i}
\end{aligned}
$$

Figure 24(a) shows the anisotropy invariant map at $x / \delta_{0}=11.5$, with an expanded view of the part near the outer edge of the boundary layer in figure 24(b). It can be seen that there are significant changes over the concave surface. The near-wall region still approaches the two-component limit, but does so further from the one-component point, consistent with less well organised near-wall velocity streaks. The maximum anisotropy occurred in the CurvR308mm case at $y / \delta_{0}=0.0084$, which falls approximately in the buffer layer. At the outer edge of the boundary layer the convex wall cases become closer to isotropic and the turbulence state spends more time close to axisymmetric compression. These characteristics are also observed in the DNS conducted by Grilli et al. 

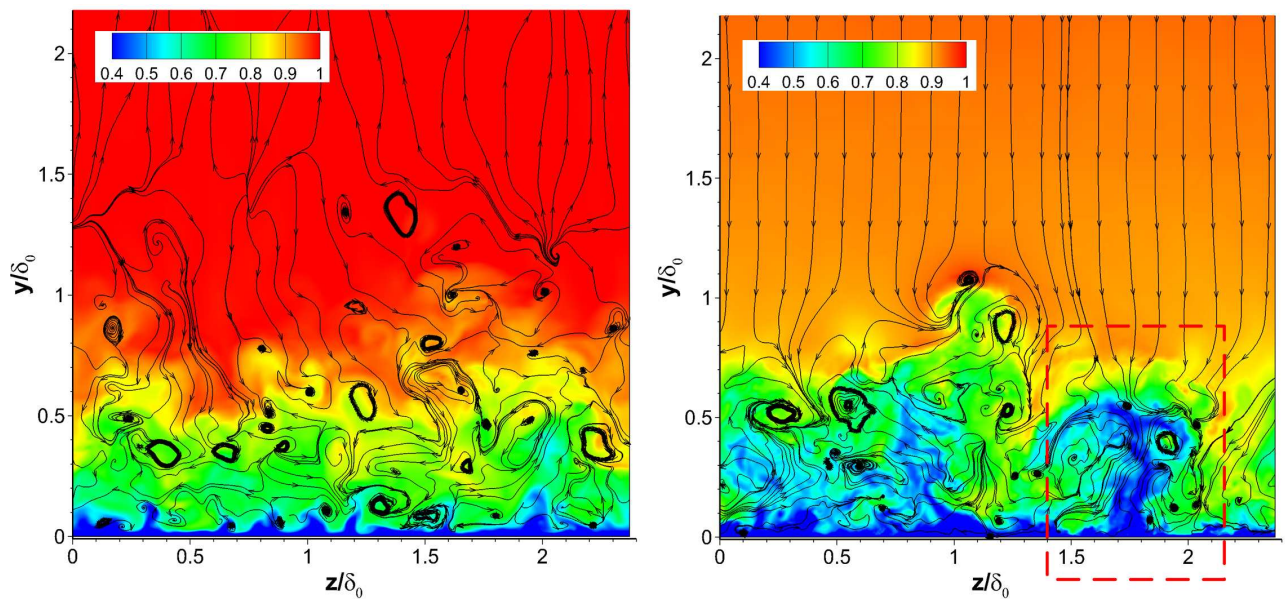

(a) Streamwise velocity $\left(u_{t} / U_{\infty}\right)$ contours, superimposed with in-plane streamlines
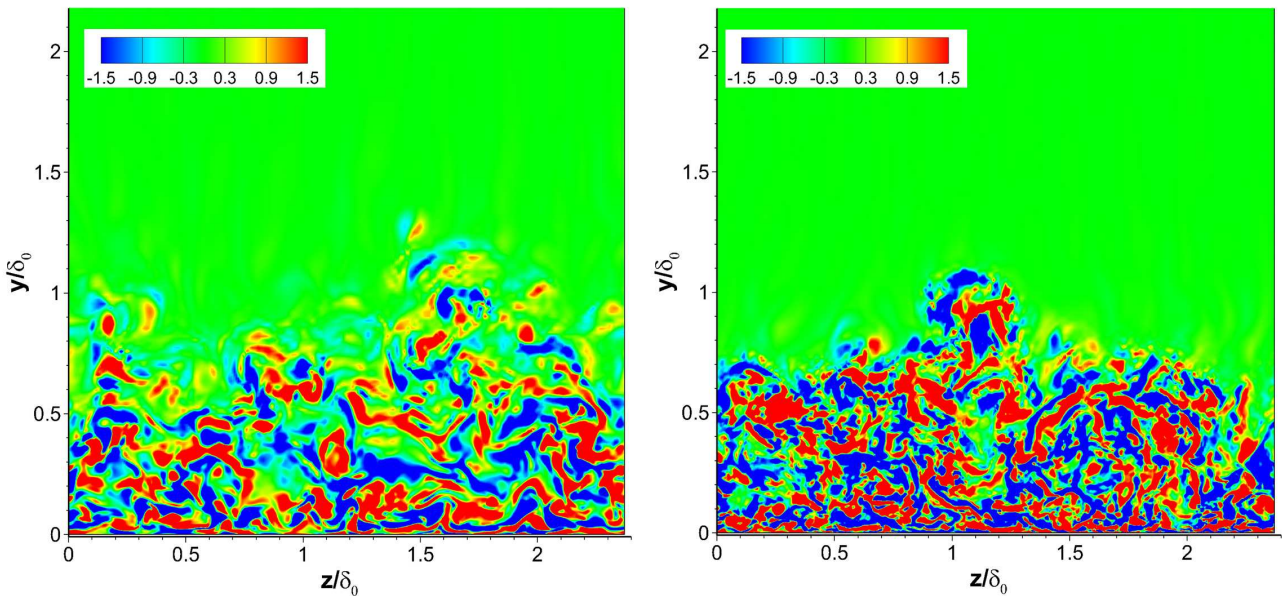

(b) Streamwise vorticity $\left(\omega_{x} /\left(U_{\infty} / \delta_{0}\right)\right)$ distribution
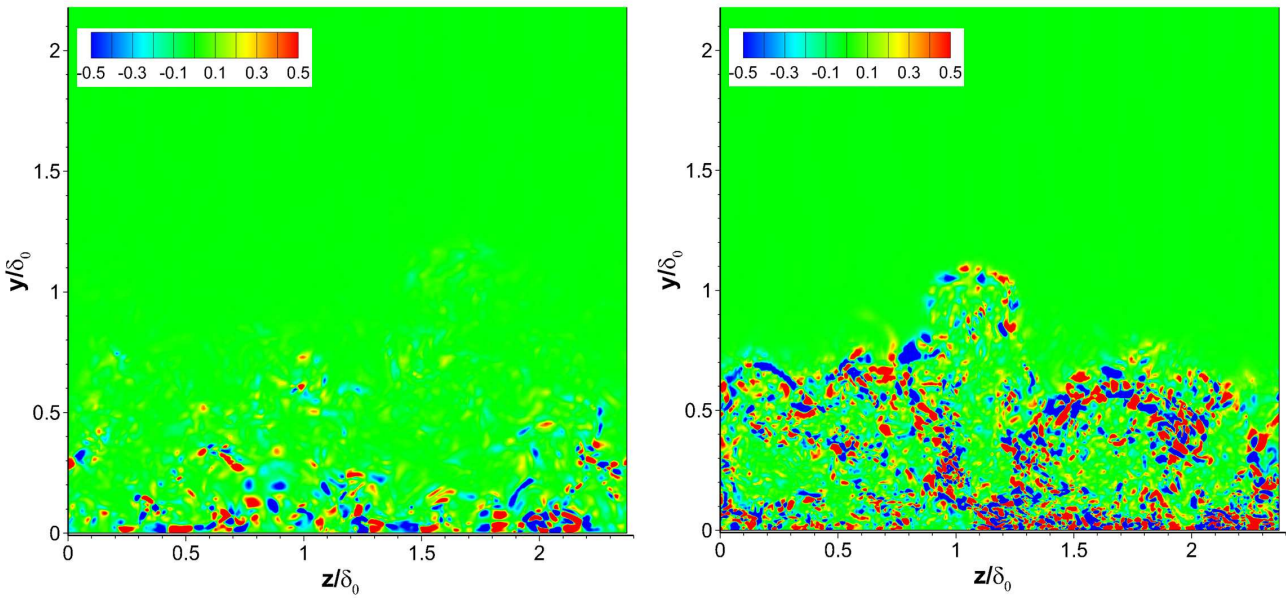

(c) Baroclinic production $\left(\left|B_{p}\right| /\left(U_{\infty}^{2}\right) / \delta_{0}^{2}\right)$

Figure 23. Streamwise velocity, vorticity and baroclinic production contours for the flat plate (left) and the CurvR308mm (right) case at $x / \delta_{0}=11.5$ 


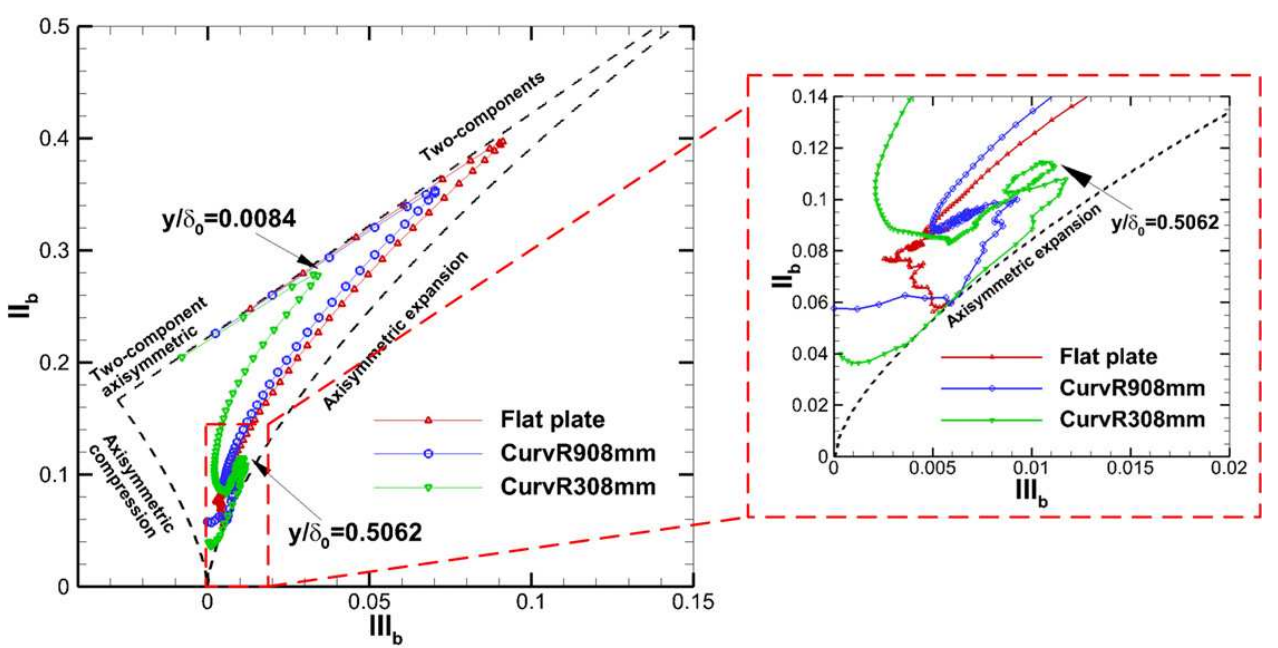

(a)

(b)

FiguRE 24. Anisotropy invariant maps based on $\left(I I I_{b}, I I_{b}\right)$ and $(\Psi, \varphi)$ respectively at $x / \delta_{0}=11.5$ for different cases (a) overall and (b) enlarged view

(2013) and Tong et al. (2017). Based on the comparison with Fig. 11-12, the anisotropy results are in accordance with the two-layer structure of the concave boundary layer.

It is difficult to extract Görtler-like vortices from the chaotic background shown in figure 23 (a)-(b), and this is probably the reason why no experiments have found Görtlerlike vortices under turbulent conditions. Ren \& Fu (2015b) used instability analysis to show the nonlinear development of Görtler vortices and the formation of low and high speed streaks in a laminar supersonic boundary layer at Mach 3.0. Their results showed that a mushroom structure is developed in the flowfield. As mentioned above, there is no regular Görtler structure in the current turbulent flow where small vortices stir and break down the large scales. However, the structures showed up clearly in the streamline plots of Figure 22. It is believed that the Görtler vortices feed the generation of large numbers of small vortices and significantly promote the exchange of the inner layer with the outer layer. On the concave wall, the inner region is mainly affected by the adverse pressure gradient and the local turbulence amplification can be explained by previous research (Lee \& J.Sung 2009; Franko \& Lele 2014) on flat plate supersonic boundary layers. As previously noted, the adverse pressure gradient interacts with the density gradients formed around streaks in the inner layer, and the resulting baroclinic effects lead to the formation of many small vortices, as seen in figure 19.

Figure 25 summarizes the role of Görtler instabilities in the turbulent supersonic concave boundary layer. A schematic view on the Görtler vortices is shown in figure 25(a), which shows a typical mushroom shape and the associated modulation of the inner boundary layer. The important result is that Görtler vortices twist and distort the local density gradient. Figure 25(b) shows the inner region flow interaction with adverse pressure gradient, which also represents the boundary layer interaction with the adverse pressure gradient without centrifugal effects. As given in the previous studies (Fernando \& Smits 1990; Donovan et al. 1994; Flaherty \& Austin 2013b; Wang et al. $2016 a$ ), the boundary layer thickness on the flat plate decreases when subject to adverse pressure gradient. Comparing figure 25(a)-(b), the prominent difference is that the Görtler vortices induce stretching, twisting and distortion of the density gradient surfaces 


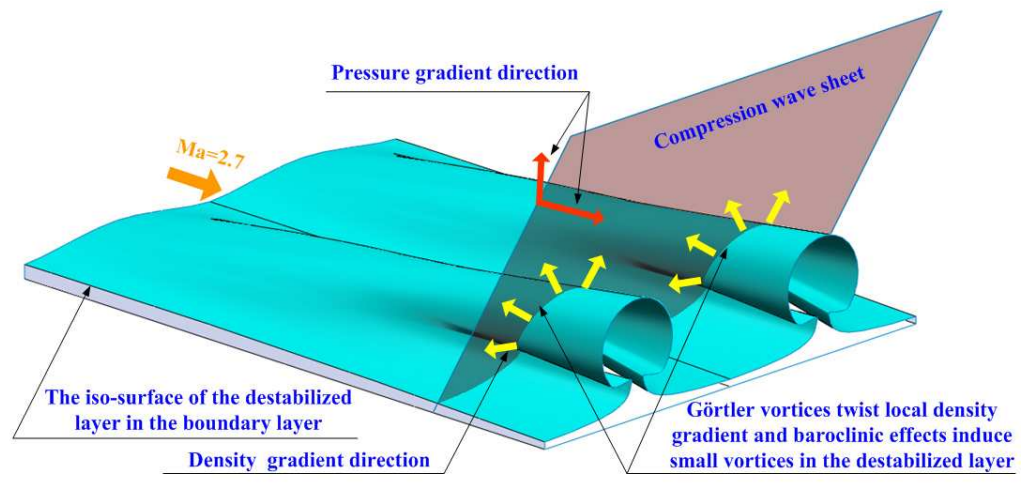

(a) Görtler instabilities in the outer portion of the boundary layer twist local gradient

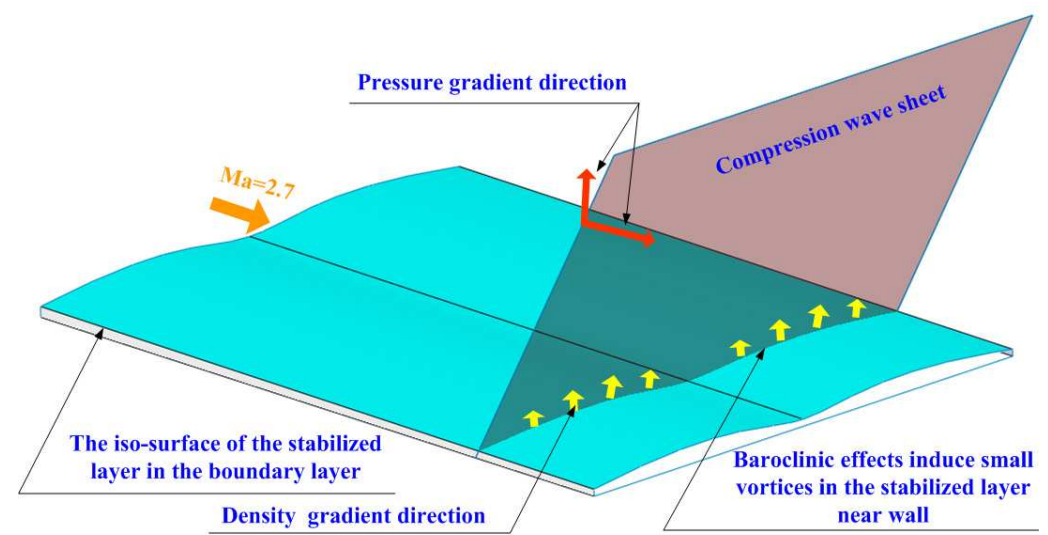

(b) Baroclinic effects on the boundary layer experiencing an adverse pressure gradient

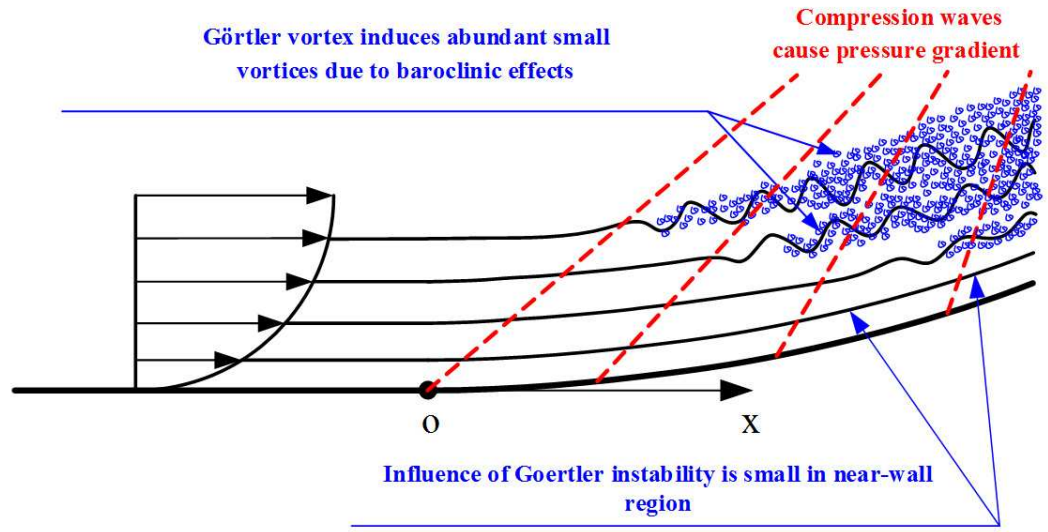

(c) Görtler vortices promote exchange process in the outer boundary layer

FIGURE 25. Schematic of Görtler instabilities in a supersonic concave boundary layer and the small vortices formed by the large baroclinic production induced by the density gradient from Görtler-like vortices and the pressure gradient from the concave compression 
to intersect with compression wave sheet, which generate larger baroclinic effect to induce more vorticities than on the flat plate. Figure 25(c) shows that the compression waves interact with the Görtler vortices and induce abundant small vortices. It might be argued that incompressible flow on concave walls would also contain Görtler vortices and a turbulence augmentation through vortex breakdown. However, a favorable pressure gradient and a uniform density for incompressible flow on concave walls would slow down the turbulence production and even lead to relaminarization(Fernholz \& Warnack 1998). For the supersonic boundary layer, favourable pressure gradients similarly weaken the coherent structures (Spalart \& Watmuff 1993; Tichenor et al. 2013; Sun et al. 2017) and lead to a turbulence suppression in the outer region of the boundary layer. For the supersonic concave boundary layer, as found by Kim et al. (2001), the turbulence amplification was closely related to the linear increase in the mean density during the supersonic compression. It could be inferred that baroclinic effects are important and seem to be fundamental for the formation of small vortices and the corresponding turbulence augmentation. In the next section we will give more quantitative results based on TKE budgets.

\section{Turbulence intensity and TKE analysis}

\subsection{TKE distribution}

Figure 26 compares the RMS values of all three velocity components and the Reynolds stress $\left.\left(<-u^{\prime} v^{\prime}\right\rangle\right)$ on the flat plate and in the CurvR308mm case at different streamwise locations. On the concave wall, velocity fluctuations for all components are amplified. Just downstream of the turning point, the turbulence level increases slightly. Whereas the inner layer peaks are almost unchanged, in the outer layer turbulence is significantly augmented along the concave wall, and the magnitudes of all velocity fluctuations are larger than those of the flat plate case.

The TKE is defined as $\tilde{k}=\overline{\rho u_{i}^{\prime \prime} u_{i}^{\prime \prime}} / 2 \bar{\rho}$, where the superscript refers to fluctuations from the Favre averages. TKE profiles at different streamwise locations of various cases are shown in figure 27. As expected, significant amplification of the TKE occurs on the concave surface. Turbulence in the inner layer of the boundary layer (i.e. the inner peak at $y^{+}=12$ ) increases downstream of the turning point compared to the same location on the flat plate, but then decreases along the concave wall. By contrast, the outer layer of the boundary layer experiences strong turbulence amplification and an outer peak emerges. A smaller curvature radius (CurvR308mm) results in a larger amplification across the boundary layer, shown in figure 27(c). Therefore, a strong two-layer structure of turbulence can be identified in the concave region. The structure is attributed to the turbulence shear stress amplification in the boundary layer on the concave wall, presumed to be due to the large-scale Görtler structures, while the inner boundary layer remains in local equilibrium. The phenomenon of the outer peak formation is similar to the second outer peak occurring in high Reynolds number wall flow. Vallikivi et al. $(2015 a, b)$ showed that for the low Reynolds numbers there is no TKE peak in the outer layer of zero pressure gradient boundary layer, while for very high Reynolds number, a peak emerges in the outer layers. Smits et al. (2011) summarized researches on high Reynolds number $\left(46,700 \leqslant R e_{\theta} \leqslant 235,000\right)$ wall turbulence and indicated that very-large-scale motions (VLSM) make a significant contribution to the turbulent kinetic energy and Reynolds stress production in the logarithmic and outer layer. This is relevant here since Görtler vortices play an important role in turbulence amplification for concave flows, whereas 


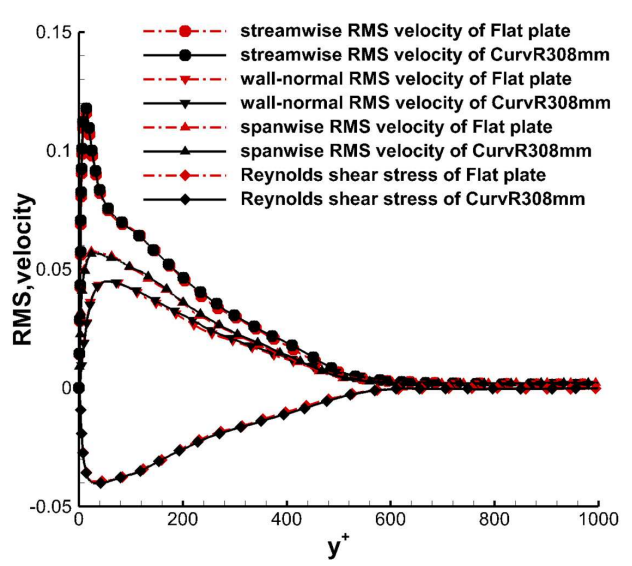

(a)

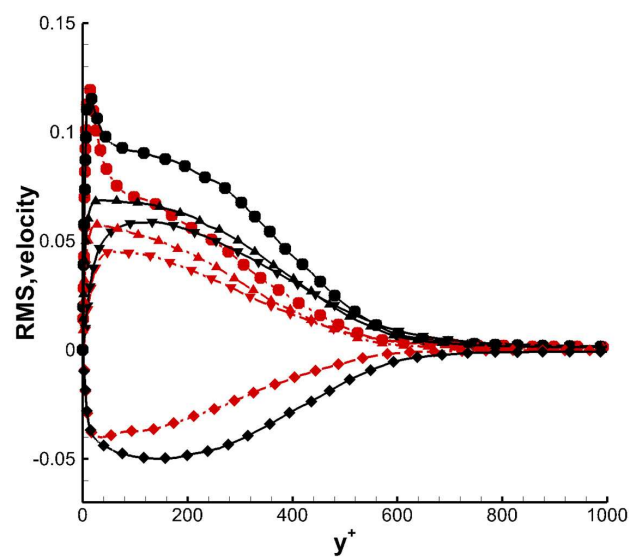

(c)

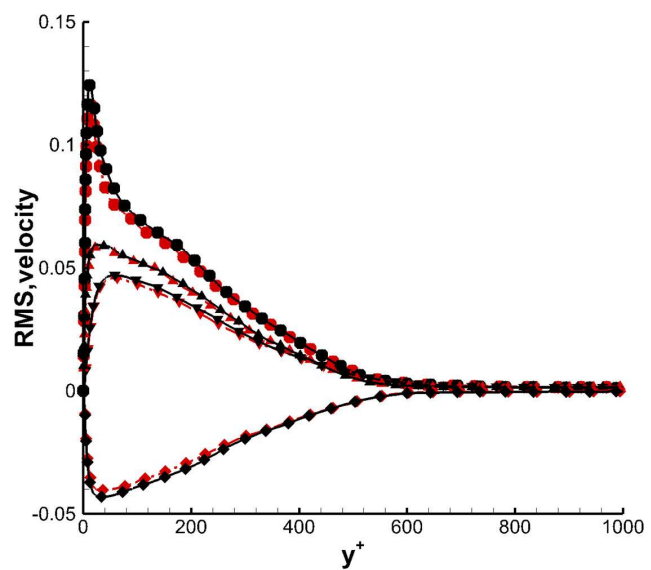

(b)

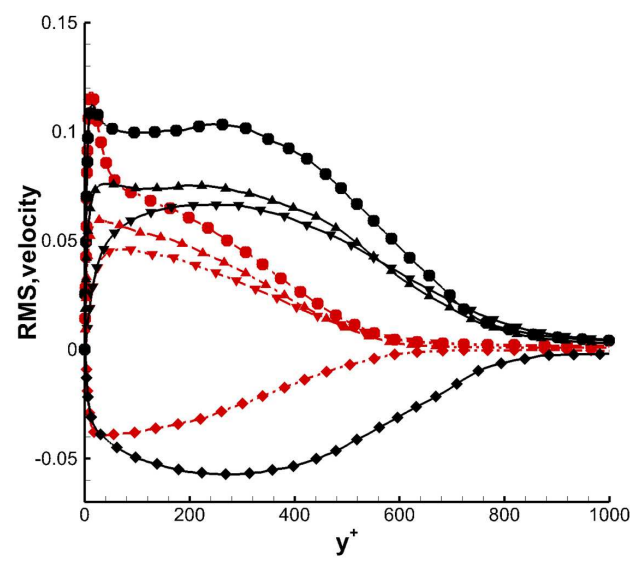

(d)

FIGURE 26. RMS velocity distributions at different streamwise locations, normalized by freestream velocity. Solid black lines represent the CurvR308mm case, Dash-dotted red lines represent the flat plate case, RMS velocity of the streamwise $(\bullet)$, wall-normal $(\mathbf{\nabla})$, spanwise $(\mathbf{\Lambda})$, of velocity fluctuations, together with Reynolds shear stress ( ), (a) $x / \delta_{0}=-2.42$, (b) $x / \delta_{0}=0.81$, (c) $x / \delta_{0}=5.65$, (d) $x / \delta_{0}=10.50$

for high Reynolds number wall turbulence, the origin of the VLSMs is still a challenging problem (Smits et al. 2011).

\subsection{TKE budgets}

The evaluation of budgets of the TKE mainly focuses on the following terms. The explicit forms of the different terms are given in Guarini et al. (2000); Sun et al. (2017) and briefly listed below,

$$
\frac{\partial}{\partial t}(\bar{\rho} \bar{k})+\tilde{u}_{j} \frac{\partial}{\partial x_{j}}(\bar{\rho} \bar{k})=P+T+I I+D-\phi+V_{c}
$$

where

$$
P=-\overline{\rho u_{i}^{\prime \prime} u_{j}^{\prime \prime}} \frac{\partial \tilde{u}_{i}}{\partial x_{j}}
$$


30
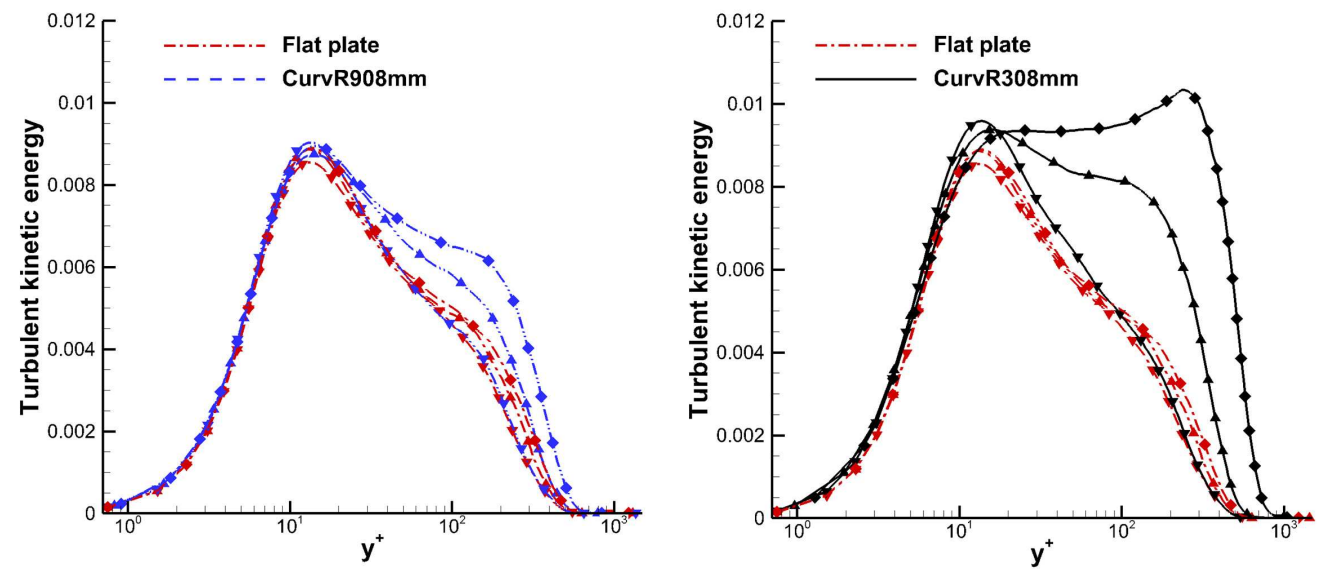

(a) Comparison of flat plate and CurvR908mm (b) comparison of flat plate and CurvR308mm case case

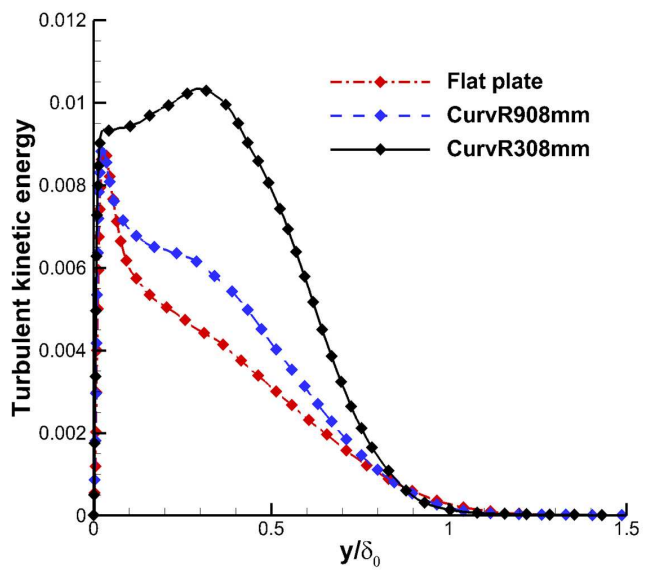

(c) comparison of flat plate, CurvR908mm case and CurvR308mm case

FIgURE 27. Turbulence kinetic energy profile comparison of various cases at different streamwise locations, (a) comparison between flat plate and CurvR908mm case at $x / \delta_{0}=0.81$ (symbol $\mathbf{\nabla}$ ), $x / \delta_{0}=5.65$ (symbol $\mathbf{\Lambda}$ ), and $x / \delta_{0}=10.50$ (symbol $\downarrow$ ), (b) comparison between flat plate and CurvR308mm case at $x / \delta_{0}=0.81$ (symbol $\mathbf{\nabla}$ ), $x / \delta_{0}=5.65$ (symbol $\mathbf{\Delta}$ ), and $x / \delta_{0}=10.50$ (symbol -), (c) comparison between flat plate, CurvR908mm case and CurvR308mm case at $x / \delta_{0}=15.35$ (symbol $\downarrow$ ). Dash-dotted line represents flat plate case, Dashed line represents CurvR908mm case, Solid line represents CurvR308mm case

$$
\begin{gathered}
T=-\frac{1}{2} \frac{\partial}{\partial x_{j}} \overline{\rho u_{i}^{\prime \prime} u_{i}^{\prime \prime} u_{j}^{\prime \prime}} \\
I I=I I_{t}+I I_{d}=-\frac{\partial}{\partial x_{j}} \overline{p^{\prime} u_{i}^{\prime \prime}} \delta_{i j}+\overline{p^{\prime} \frac{\partial u_{i}^{\prime \prime}}{\partial x_{i}}} \\
D=-\frac{\partial}{\partial x_{j}} \overline{\frac{u_{i}^{\prime \prime}}{\operatorname{Re}} \tau_{i j}^{\prime}} \\
\phi=\frac{1}{\operatorname{Re} \frac{\partial u_{i}^{\prime \prime}}{\partial x_{j}} \tau_{i j}^{\prime}}
\end{gathered}
$$




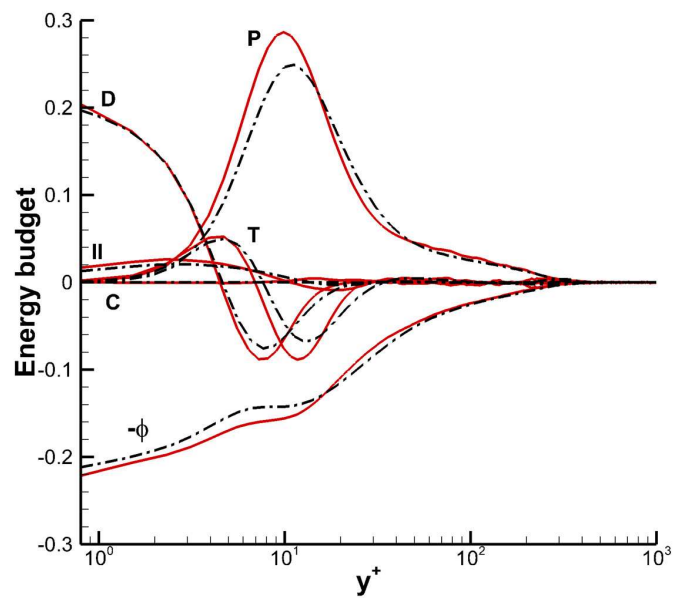

Figure 28. TKE budget at $x / \delta_{0}=0$ at the current flat plate case. Dashdotted line represents results of the incompressible results of Schlatter \& Örlü (2010) at Reynolds number of $\operatorname{Re}_{\theta}=1,000$, solid line represents the current flat plate case.

$$
\begin{gathered}
V_{c}=-\overline{u_{j}^{\prime \prime}} \frac{\partial \bar{p}}{\partial x_{j}}+\frac{\overline{u_{i}^{\prime \prime}}}{\operatorname{Re}} \frac{\partial \overline{\tau_{i l}}}{\partial x_{l}}-\bar{\rho} \bar{k} \frac{\partial \tilde{u}_{j}}{\partial x_{j}} \\
C=-\tilde{u}_{j} \frac{\partial}{\partial x_{j}}(\bar{\rho} \bar{k})
\end{gathered}
$$

Here, $P$ is the production term, showing the rate of generation of TKE by mean velocity gradients, $T$ is the turbulent transport, II is the pressure diffusion and dilatation, $D$ is the viscous diffusion, $\phi$ is the viscous dissipation and $C$ is the convective term. $V_{c}$ includes the terms that arise when the density is not constant. Compared to other terms $V_{c}$ is small, therefore has not been included on the plot for clarity. Its maximum value is a factor of 28 and 26 smaller than that of the production term in the present calculation in the flat plate case and CurvR308mm case respectively.

We firstly compare the TKE budgets at $x / \delta_{0}=0$ of the flat plate case, where the corresponding Reynolds number, based on the boundary-layer momentum thicknesses $\theta_{0}=0.48 \mathrm{~mm}$, under the van Driest scaling is approximately $R e_{\theta_{0}}=1078$. All terms are normalised by the wall quantity $\rho_{w} u_{\tau}^{4} / \nu_{w}$. All terms balance each other, and their sum is no more than $10^{-3}$. Figure 28 compares the distributions of these terms with the incompressible results of Schlatter \& Örlü (2010) at Reynolds number $R_{\theta}$ of 1000 , with the results consistent with the incompressible simulations. The simulation data is also similar to results of Guarini et al. (2000). As Guarini et al. (2000) and Pirozzoli et al. (2004) pointed out, the agreement between the supersonic flow on flat plate and the incompressible flow demonstrates the validity of Morkovins hypothesis (Morkovin 1962), which means the compressibility terms are negligible throughout a spatially evolving supersonic turbulent boundary layer on the flat plate. Lagha et al. (2011) and Duan \& Beekman (2011) studied Mach number effects on a hypersonic boundary layer at zero pressure gradient and showed that the weak compressibility hypothesis remains valid for a large range of free-stream Mach numbers.

To further assess the boundary layer on the concave walls, TKE budgets are compared to the flat plate results at different streamwise locations, as shown in figure 29. At the location $x / \delta_{0}=-2.42$ upstream of the corner, there is no difference between the flat plate and the concave cases. TKE budgets all show behaviour typical of a boundary 


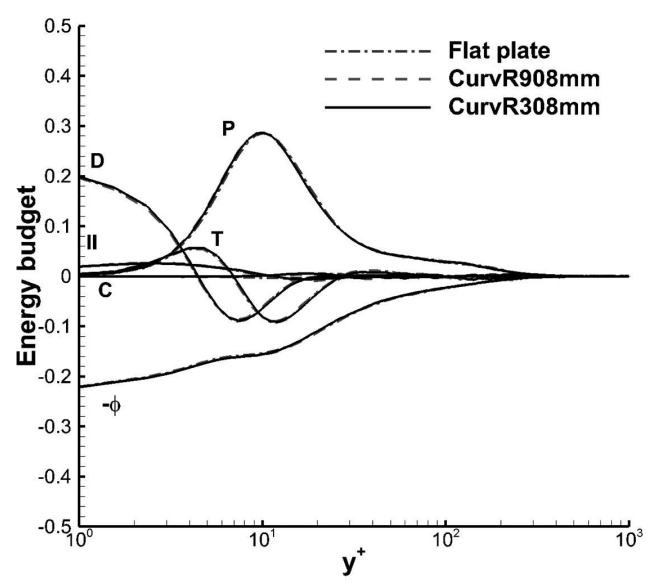

(a) $x / \delta_{0}=-2.42$

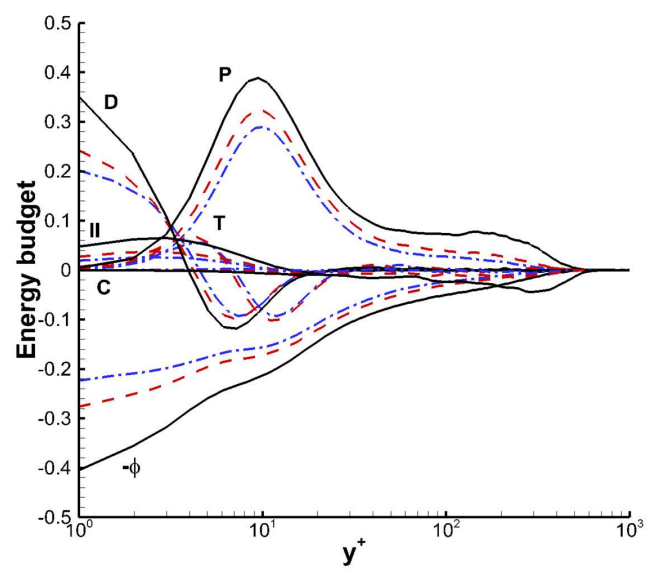

(c) $x / \delta_{0}=5.65$

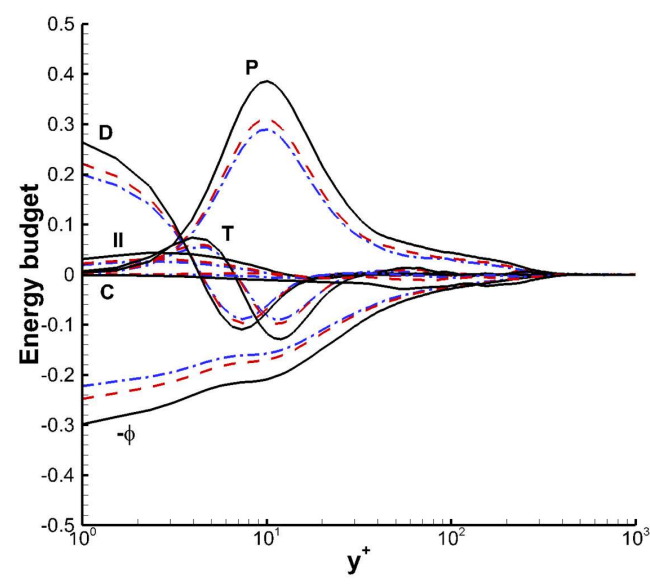

(b) $x / \delta_{0}=0.81$

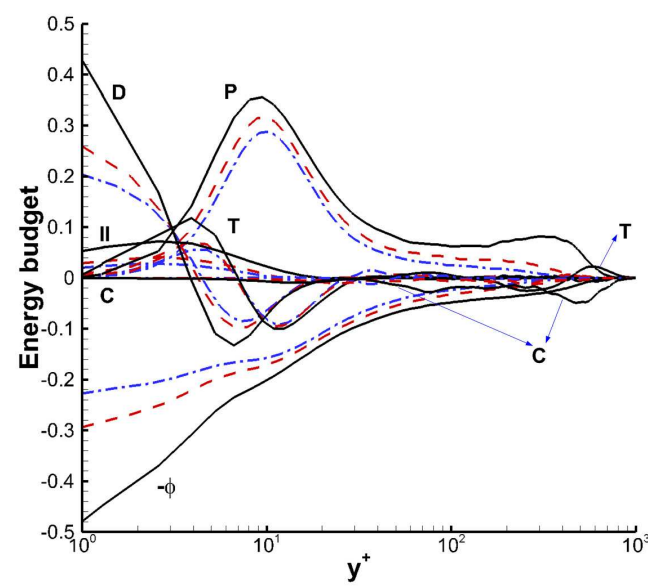

(d) $x / \delta_{0}=10.50$

Figure 29. Turbulent kinetic energy budget profiles of the flat plate case and concave cases at different streamwise locations. Dashdotted line represents the flat plate case, - Dashed line represents CurvR908mm case, solid line represents CurvR308mm case

layer at zero-pressure gradient in which production is balanced by dissipation over most of the boundary layer, as also described by Guarini et al. (2000) and Pirozzoli et al. (2004).Viscous diffusion is only important in the near wall region. In the viscous sublayer, production becomes negligible and diffusion is balanced by dissipation.

At $x / \delta_{0}=0.81$ in the turning region, compared to the flat plate case, all terms in the concave cases increase in magnitude in the inner layer. TKE production increases slightly in the outer layer $\left(y^{+} \in[60,500]\right)$. The pressure diffusion and dilatation II terms in the TKE equation become apparent due to the APG caused by the concave compression. The convection term $C$ at the boundary layer edge falls to a larger negative values, which means the convection from the boundary layer to the mainstream is augmented. TKE becomes higher from the interaction of the boundary layer with the mainstream at $x / \delta_{0}=0.81$.

At $x / \delta_{0}=5.65$, all terms in the TKE budget equation are amplified for the concave cases, while the TKE budget terms in the inner layer increase in magnitude with curvature rate. In the outer layer where $y^{+} \in[100,600]$, the dissipation increases with curvature 


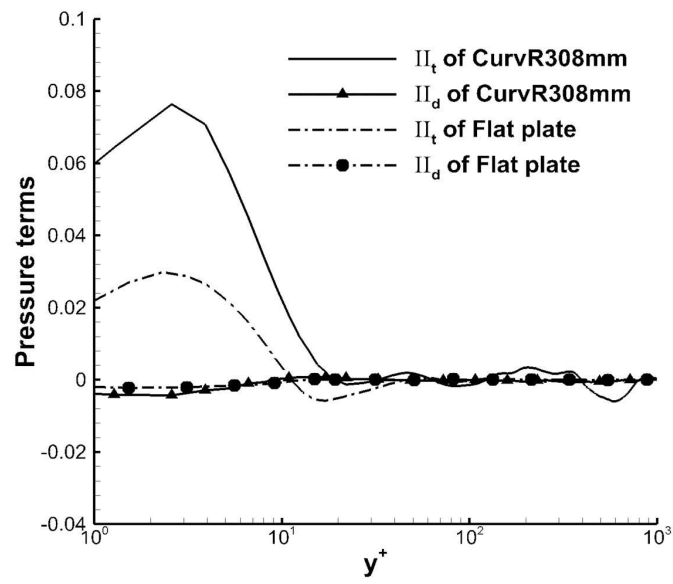

Figure 30. Comparison of pressure terms of flat plate case and CurvR308mm case at $x / \delta_{0}=10.50$

rate while TKE production and TKE convection term are amplified significantly. The production augmentation is basically balanced by a negative increment in convection, which means the turbulence produced in the outer layer is mainly convected away.

At $x / \delta_{0}=10.50$ the production term of the CurvR308mm case becomes larger than that of the CurvR908mm case, especially in the outer layer $y^{+} \in[100,1000]$. This also corresponds to the generation of the outer peak in the TKE profile for CurvR308mm case. The TKE pressure diffusion $\left(I I_{t}\right)$ and dilatation $\left(I I_{d}\right)$ term $I I$ becomes more significant due to the adverse pressure gradient caused by the concave wall. Here we consider the contribution of the pressure terms, $I I_{t}$ and $I I_{d}$. Figure 30 shows the comparison of pressure terms at $x / \delta_{0}=10.50$ between the CurvR308mm case and the flat plate case. The pressure diffusion term is larger than pressure dilatation term, which indicates that pressure diffusion effect plays a dominant role, instead of the explicit compressibility effect. The magnitude of the convection term increases significantly in the outer layer of the boundary layer while the dissipation is amplified slightly, which means local convection of turbulent energy is amplified for CurvR308mm case. The transport term is also amplified at $x / \delta_{0}=10.50$ and participates in balancing the production.

\section{Compressibility effects}

\subsection{Dilatation}

To further assess the compressibility and curvature effects, the probability distribution function (PDF) of the dilatation term, $\Theta=\partial_{x} u+\partial_{y} v+\partial_{z} w$, is computed. Figure 31 shows the PDF, which is computed by averaging over the range $y / \delta_{0} \in(0.1,0.5)$ and $x / \delta_{0} \in(0.81,10.50)$. For the flat plate case the PDF is sharp and peaks at zero dilatation. However, on the concave walls, the PDF is wider and the peak shifts to negative values of dilatation. The latter effect can be explained from the continuity equation,

$$
\frac{\partial \rho}{\partial t}+\frac{\partial \rho u_{i}}{\partial x_{i}}=\frac{\partial \rho}{\partial t}+\nabla \rho \cdot \mathbf{u}+\Theta \rho=0
$$

Since $\nabla \rho \cdot \mathbf{u}$ is always positive on the concave wall, for small fluctuations we expect the average $\Theta$ to be negative. With increasing curvature the dilatation PDF of dilatation becomes more skewed, with more extreme events of large negative dilation, which for 


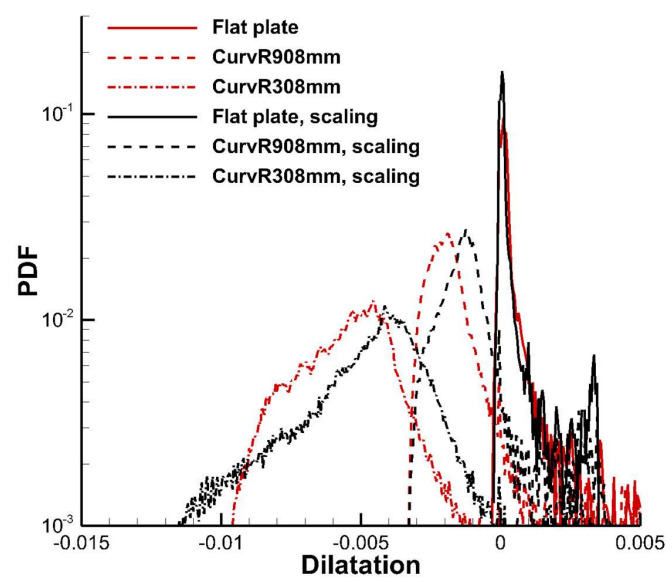

FiguRE 31 . The pdf of the dilatation is computed statistically in (x,y)-planes corresponding to different wall-normal locations $y / \delta_{0} \in(0.1,0.5)$ and $x / \delta_{0} \in(0.81,10.50)$ without scaling and with density scaling.

higher Mach numbers or stronger curvatures may lead to the formation of eddy shocklets. A scaling of pdf $\bar{\rho} \Theta$ using the mean density $\bar{\rho}$ has previously been used to collapse different PDF curves, as discussed by Lagha et al. (2011). Figure 31 includes the density scaling $\mathrm{PDF}$, however the distribution is similar to the non-scaled data for all the concave cases.

\subsection{Turbulent Mach number}

Turbulent Mach number $M_{t}=\sqrt{\overline{u_{j}^{\prime} u_{j}^{\prime}}} / \bar{c}$ profiles are shown in figure 32 (a)-(b) at different streamwise locations for $x / \delta_{0}=0.81,5.65$ and 10.50 . In addition to the turbulent Mach number, we also examine the RMS values of the local Mach number, the fluctuating Mach number $\mathrm{M}^{\prime}\left(=\left(\overline{M^{2}}-\bar{M}^{2}\right)^{1 / 2}\right)$, which is different in compressible flows. The fluctuating Mach number profiles are shown in figure 32(c)-(d). For the flat plate case, the turbulent Mach number is no more than 0.3 and its maximum peak is located near to the wall. For the concave cases, turbulent Mach number starts to build a second peak which moves away from the wall, as seen in figure 32(a)-(b). The profiles of $M_{t}$ and $M^{\prime}$ exhibit a similar character with the outer peak shown in figure 27 . In figure $32(\mathrm{c})$-(d), the maximum of the second peak of the fluctuating Mach number exceeds 0.3 which suggests that the compressibility is approaching the borderline of the Morkovin (1962).

\subsection{Effective Mach number}

An effective Mach number has also been proposed (Sandham 2016) to evaluate the compressibility effects of a turbulent boundary layer with adverse pressure gradient variation from zero to separation, given by

$$
M_{c, b l}=\frac{\Delta U_{V D}^{+}}{U_{e}^{+}} \frac{M_{\infty}}{\left(1+a_{w} / a_{\infty}\right)}=\frac{\Delta U_{V D}^{+}}{U_{e}^{+}} \frac{M_{\infty}}{\left(1+\sqrt{T_{w} / T_{\infty}}\right)}
$$

where a means the local sound speed. $\Delta U_{V D}^{+}$refers to the van Driest-transformed velocity increment in the wake region compared to log law using the van Driest profile at $y=\delta$, i.e. 


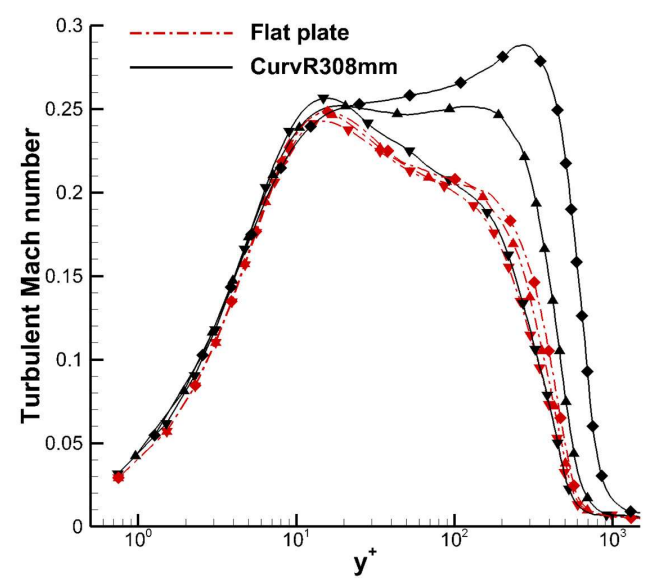

(a)

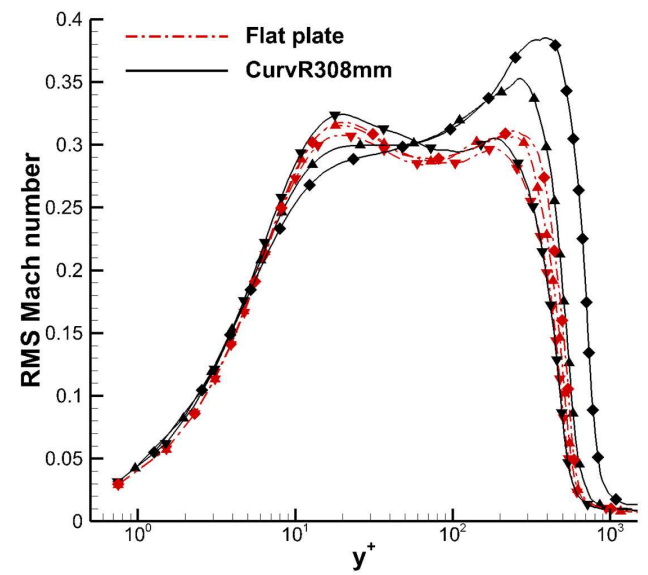

(c)

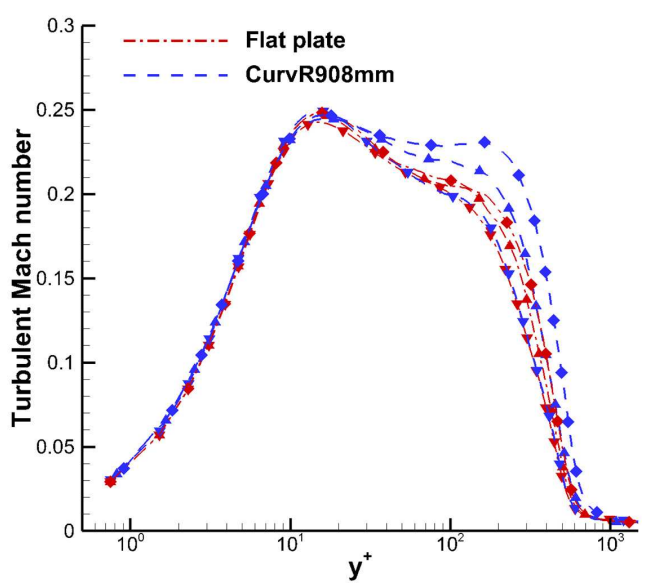

(b)

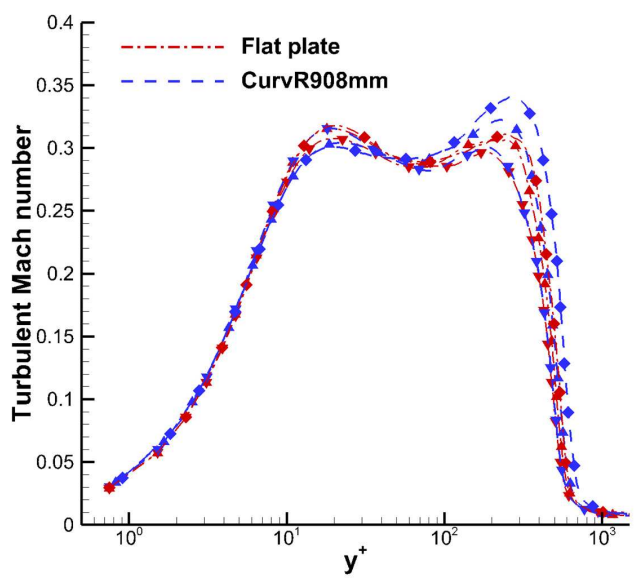

(d)

Figure 32. Profiles of the turbulent Mach number $M_{t}$ and the fluctuating Mach number $M^{\prime}$ of the flat plate and concave cases at different streamwise locations. (a) $x / \delta_{0}=0.81$ (symbol $\mathbf{\nabla}$ ), $x / \delta_{0}=5.65$ (symbol $\boldsymbol{\Delta}$ ), and $x / \delta_{0}=10.50$ (symbol $\bullet$ ), Dash-dotted line represents the flat plate case, Dashed line represents CurvR908mm case, Solid line represents CurvR308mm case.

$$
\Delta U_{V D}^{+}=U_{e, \delta}^{+}-\left(\frac{1}{\kappa} \ln \delta^{+}+b\right)
$$

where $U_{e, \delta}^{+}$means the van Driest-transformed velocity at $y=\delta$. The calculated results are shown in figure 33 . The level of 0.3 is usually taken as the threshold above which compressibility effects are expected to be significant. We see that this is not reached for the CurvR308mm case and the compressibility effect increases as curvature ratio increases. Recalling the analysis of the dilatations, turbulent Mach number and fluctuating Mach number, it is inferred that the compressibility for current concave cases does not exceed the threshold; however, for larger curvature ratios than those in this paper, the compressibility effects probably need be considered in turbulence modeling, which needs further investigations. 


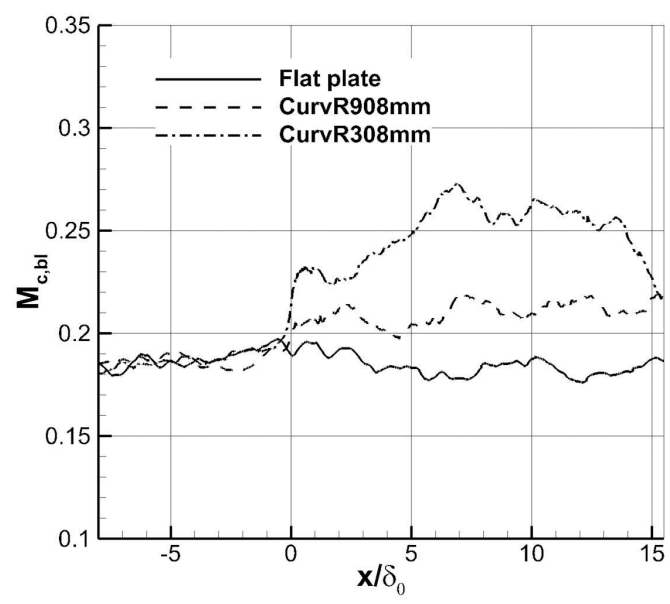

FiguRE 33. Effective Mach number $M_{c, b l}$ over flat plate and concave walls

\section{Conclusions}

Direct numerical simulations have been carried out to investigate a Mach 2.7 supersonic turbulent boundary layer over concave surfaces with curvature radius of $308 \mathrm{~mm}$ and $908 \mathrm{~mm}$, respectively. A flat plate case is also simulated for comparison.

The response of the skin friction, wall pressure and velocity profiles due to concave surface curvature is examined. Analysis of boundary layer velocity profiles shows a streamwise velocity reduction in the outer part of the boundary layer, due to compression, while an increase occurs near the wall. The simulated data suggest that compressibility effects are not negligible on concave surfaces with large curvature rate. A higher shape factor is found in the concave cases, which indicates that the fullness of the velocity profile on concave walls is reduced. Prominent low frequency fluctuations on concave walls reflect the generation of large scale structures. The streaks shown in the wall-parallel slices and the two-point correlation function demonstrate that concave effects reduce the near-wall spanwise streak spacing while producing large scale streaks in the outer layer. $\lambda_{2}$ vortex structures indicate turbulence amplification on the concave wall.

The streamwise velocity iso-surface, streamlines and contour slices show that higher speed or smaller concave curvature radius tends to destabilize local turbulent structures in the boundary layer. Görtler-like vortices generated by the centrifugal effects occur in the outer region of the concave boundary layer. The Görtler-like structures twist local density gradient and the baroclinic production from the interaction of the density gradients with the concave compression leads to the generation of abundant small scale vortices on the concave walls, which represent the amplification of local turbulence on the concave walls. The inner region of the concave boundary layer appears to be little affected by the Görtler instability and acquires a relatively slow turbulence amplification by the baroclinic effects from the adverse pressure gradient.

Profiles of TKE and turbulent Mach number along the streamwise direction exhibit a characteristic two-layer structure in the boundary layer of concave cases. In the outer layer, the turbulence is greatly amplified and the Görtler vortices lead to a rapid exchange between the inner and outer layers. Turbulent energy budget analysis shows that both production and dissipation increase near the wall in the concave region, but in the outer part of the boundary layer, the production is significantly amplified and balanced by convection and transport. 
In the future, it would be interesting to explore further the sensitivity of these results to the key parameters, including higher Reynolds number, higher Mach number and larger curvature ratios as well as range of wall thermal conditions.

\section{Acknowledgement}

Supercomputer time provided by the UK Engineering and Physical Sciences Research Council under the project Thermal and Reactive Flow Simulation on High-End Computers (Grant No. EP/J016381/1) is gratefully acknowledged.

This work was also supported by grants from the National Natural Science Foundation of China (11472305, 91541101 and 11522222), and an outstanding youth fund of National University of Defense Technology.

Data supporting this study are openly available from the University of Southampton repository at https://doi.org/10.5258/SOTON/D2436.

\section{REFERENCES}

Bradshaw, P. 1974 The effect of mean compression or dilatation on the turbulence structure of supersonic boundary layers. J. Fluid Mech. 63, 449-466.

Ciolkosz, L. \& Spina, E. 2006 An experimental study of Görtler vortices in compressible flow. AIAA Paper 2006-4512.

Donovan, J. F., Spina, E. F. \& Smits, A. J. 1994 The structure of a supersonic turbulent boundary layer subjected to concave surface curvature. J. Fluid Mech. 259, 1-24.

Duan, L. \& Beekman, I. 2011 Direct numerical simulation of hypersonic turbulent boundary layers. part 3. effect of mach number. J. Fluid Mech. 672 (4), 245-267.

Ducros, F., Ferrand, V., Nicoud, F., Weber, C., Darracq, D., Gacherieu, C. \& Poinsot, T. 1999 Large-eddy simulation of the shock/turbulence interaction. J.comput.phys 152 (2), 517-549.

Fernando, E. M. \& Smits, A. J. 1990 A supersonic turbulent boundary layer in an adverse pressure gradient. J. Fluid Mech. 211, 285-307.

Fernando, E. M. \& Smits, A. J. 2006 A supersonic turbulent boundary layer in an adverse pressure gradient. J. Fluid Mech. 211, 285-307.

Fernholz, H. H. \& WARnACK, D. 1998 The effects of a favourable pressure gradient and of the reynolds number on an incompressible axisymmetric turbulent boundary layer. part 1. the turbulent boundary layer. J. Fluid Mech. 359, 329-356.

Flaherty, W. \& Austin, J. M. 2013 a Scaling of heat transfer augmentation due to mechanical distortions in hypervelocity boundary layers. Phys. Fluids 25, 106-106.

Flaherty, W. \& Austin, J. M. $2013 b$ Scaling of heat transfer augmentation due to mechanical distortions in hypervelocity boundary layers. Physics of Fluids 25 (10), 379-409.

Flaherty, W. P. 2013 Effects of Local and Global Mechanical Distortions to Hypervelocity Boundary Layers. University of Illinois at Urbana-Champaign.

Franko, K. J. \& Lele, S. 2014 Effect of adverse pressure gradient on high speed boundary layer transition. Physics of Fluids 26 (2), 176-183.

GöRTLER, H. 1954 On the three-dimensional instability of laminar boundary layers on concave walls. Tech. Rep. 1375. NASA Tech. Mem.

Green, J. E. 1970 Interactions between shock waves and turbulent boundary layers. Prog. Aerosp. Sci. 11, 235-340.

Grilli, M., Hickel, S. \& AdAms, N. A. 2013 Large-eddy simulation of a supersonic turbulent boundary layer over a compressioncexpansion ramp. International Journal of Heat Fluid Flow 42 (8), 79-93.

Guarini, S. E., Moser, R. D., Shariff, K. \& Wray, A. 2000 Direct numerical simulation of a supersonic turbulent boundary layer at mach 2.5. J. Fluid Mech. 414, 1-33.

Harun, Z., Monty, J. P., Mathis, R. \& Marusic, I. 2013 Pressure gradient effects on the large-scale structure of turbulent boundary layers. J. Fluid Mech. 715, 477-498. 
Hu, Z. W., Morfey, C. L. \& D.Sandham, N. 2006 Wall pressure and shear stress spectra from direct numerical simulations of channel flow. AIAA Journal 44(7), 1541-1549.

JeOng, J. \& Hussain, F. 1995 On the identification of a vortex. J. Fluid Mech. 285, 69-94.

Kim, J., Samimy, M. \& LeE, S. 2001 Effects of compression and expansion on turbulence intensity in supersonic boundary layers 39, 1071-1077.

Lagha, M., Kim, J., Eldredge, J. D. \& Zhong, X. 2011 A numerical study of compressible turbulent boundary layers. Physics of Fluids 23 (1), 187.

LEe, J. H. \& J.Sung, H. 2009 Structures in turbulent boundary layers subjected to adverse pressure gradients. J. Fluid Mech. 639, 101-131.

Li, F., Choudhari, M., Chang, C.-L., WU, M. \& Greene, P. T. 2010 Development and breakdown of Görtler vortices in high speed boundary layers. AIAA Paper 2010-705 .

Luca, L., Cardone, G., Aymer de la Chevalerie, D. \& Fonteneau, A. 1993 Goertler instability of a hypersonic boundary layer. Exp. Fluids 16, 10-16.

Luker, J. J., Hale, C. S. \& Bowersox, R. D. W. 1998 Experimental analysis of the turbulent shear stresses for distorted supersonic boundary layers. J. Propul. Power 14, 110-118.

Lumley, J. L. 1978 Computational modeling of turbulent flows. Archive of Applied Mechanics 18, 123-176.

Maeder, T., Adams, N. A. \& Kleiser, L. 1998 Direct Numerical Simulation of Supersonic Turbulent Boundary Layers. Springer Netherlands.

Morkovin, M. V. 1962 Effects of compressibility on turbulent flows. In Mecanique de la Turbulence (ed. A. Favre) p. 367C380. CNRS.

Neel, I., Leidy, A. N., Bowersox, R. D. W. \& Tichenor, N. R. 2016 Hypersonic boundary layer with streamline curvature-driven adverse pressure gradient. AIAA Paper 2016-4248

Pirozzoli, S., Grasso, F. \& Gatski, T. B. 2004 Direct numerical simulation and analysis of a spatially evolving supersonic turbulent boundary layer at $\mathrm{m}=2.25$. J. Fluid Mech. 16, $530-545$.

REN, J. \& FU, S. 2015 a Secondary instabilities of Görtler vortices in high-speed boundary layer flows. J. Fluid Mech. 781, 388-421.

Ren, J. \& Fu, S. $2015 b$ Study of the discrete spectrum in a mach 4.5 Görtler flow. Flow Turbul. Combust. 94, 339-357.

SANDhAM, N. D. 2016 Effects of compressibility and shock-wave interactions on turbulent shear flows. Flow Turbul. Combust. 97, 1-25.

Sandham, N. D., Schülein, E., Wagner, A., Willems, S. \& Steelant, J. 2014 Transitional shock-wave/boundary-layer interactions in hypersonic flow. J. Fluid Mech. 752, 349-382.

SARIC, W. S. 1994 Görtler vortices. Annu. Rev. Fluid Mech. 26, 379-409.

Schlatter, P. \& ÖRlü, R. 2010 Assessment of direct numerical simulation data of turbulent boundary layers. J. Fluid Mech. 659, 116-126.

Sмiтh, D. R. \& Smits, A. J. 1994 The effects of steamline curvature and pressure gradient on the behavior of turbulent boundary layers in supersonic flow. AIAA Paper 94-2227.

Sмith, D. R. \& Smits, A. J. 1995 A study of the effects of curvature and compression on the behavior of a supersonic turbulent boundary layer. Exp. Fluids 18, 363-369.

Smits, A. J. \& Dussauge, J.-P. 2006 Turbulent Shear Layers in Supersonic Flow, 2nd edn. Springer.

Smits, A. J., McKeon, B. J. \& Marusic, I. 2011 High-Reynolds number wall turbulence. Annu. Rev. Fluid Mech. 43, 353-375.

Spalart, P. R. \& Watmuff, J. H. 1993 Experimental and numerical study of a turbulent boundary layer with pressure gradients. J. Fluid Mech. 249, 337-371.

Spina, E. F., Smits, A. J. \& Robinson, S. K. 1994 The physics of supersonic turbulent boundary layers. Annu. Rev. Fluid Mech. 26, 287-319.

Sun, M. B. \& Hu, Z. W. 2018a Formation of surface trailing counter-rotating vortex pairs downstream of a sonic jet in a supersonic cross-flow. J. Fluid Mech. 850, 551-583.

Sun, M. B. \& Hu, Z. W. $2018 b$ Generation of upper trailing counter-rotating vortices of a sonic jet in a supersonic crossflow. AIAA J. 56(3), 1047-1059.

Sun, M. B. \& Hu, Z. W. 2018c Mixing in nearwall regions downstream of a sonic jet in a supersonic crossflow at mach 2.7. Physics of Fluids 30, 106102. 
Sun, M. B., Hu, Z. W. \& Sandham, N. D. 2017 Recovery of a supersonic turbulent boundary layer after an expansion corner. Physics of Fluids 29 (7), 076103.

Sun, M. B., Zhang, S. P., Zhao, Y. X. \& Liang, J. H. 2013 Experimental investigation on transverse jet penetration into a supersonic turbulent crossflow. Sci. China Technol. Sci. 56, 1989-1998.

Thompson, K. W. 1990 Time dependent boundary conditions for hyperbolic systems. J.comput.phys 89 (2), 439-461.

Tichenor, N. R., Humble, R. A. A. \& Bowersox, R. D. W. 2013 Response of a hypersonic turbulent boundary layer to favourable pressure gradients. J. Fluid Mech. 722, 187-213.

Tong, F., Li, X., DuAn, Y.\& Yu, C. 2017 Direct numerical simulation of supersonic turbulent boundary layer subjected to a curved compression ramp. Physics of Fluids 29, 125101.

Touber, E. 2010 Unsteadiness in shock-wave/boundary-layer interactions. PhD thesis, University of Southampton.

Touber, E. \& SAndham, N. D. 2009 Large-eddy simulation of low-frequency unsteadiness in a turbulent shock-induced separation bubble. Theor. Comput. Fluid Dyn. 23, 79-107.

Vallikivi, M., Ganapathisubramani, B. \& Smits, A. J. 2015 a Spectral scaling in boundary layers and pipes at very high Reynolds numbers. J. Fluid Mech. 771, 303-326.

Vallikivi, M., Hultmark, M. \& Smits, A. J. $2015 b$ Turbulent boundary layer statistics at very high Reynolds number. J. Fluid Mech. 779, 371-389.

Wang, B., Sandham, N. D., Hu, Z. W. \& LiU, W. 2015 Numerical study of oblique shockwave/boundary-layer interaction considering sidewall effects. J. Fluid Mech. 767, 526-561.

WAng, Q. C. \& WANG, Z. G. 2016 Structural characteristics of the supersonic turbulent boundary layer subjected to concave curvature. Appl. Phys. Lett. 108, 114102.

WANG, Q. C., Wang, Z. G. \& ZhaO, Y.X. 2016a An experimental investigation of the supersonic turbulent boundary layer subjected to concave curvature. Physics of Fluids 28 (9), 096104.

WAnG, Q. C., WAnG, Z. G. \& ZhaO, Y.X. $2016 b$ On the impact of adverse pressure gradient on the supersonic turbulent boundary layer. Physics of Fluids 28 (11), 116101.

White, F. M. 2007 Viscous Fluid Flow, 3rd edn. McGraw-Hill.

XIE, Z. \& CAStro, I. P. 2008 Efficient generation of inflow conditions for large eddy simulation of street-scale flows. Flow Turbul. Combust. 81, 449-470.

Yee, H.C, Sandham, N.D \& DJomenri, M.J 1999 Low-dissipative high-order shock-capturing methods using characteristic-based filters. J.comput.phys 150 (1), 199-238. 